NASA/TM-1999-209042

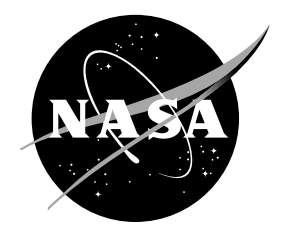

\title{
Surface Segregation in Multicomponent Systems: Modeling of Surface Alloys and Alloy Surfaces
}

Guillermo Bozzolo

Ohio Aerospace Institute, Cleveland, Ohio

John Ferrante

Cleveland State University, Cleveland, Ohio

Ronald D. Noebe, Brian Good, Frank S. Honecy, and Phillip Abel Glenn Research Center, Cleveland, Ohio 
Since its founding, NASA has been dedicated to the advancement of aeronautics and space science. The NASA Scientific and Technical Information (STI) Program Office plays a key part in helping NASA maintain this important role.

The NASA STI Program Office is operated by Langley Research Center, the Lead Center for NASA's scientific and technical information. The NASA STI Program Office provides access to the NASA STI Database, the largest collection of aeronautical and space science STI in the world. The Program Office is also NASA's institutional mechanism for disseminating the results of its research and development activities. These results are published by NASA in the NASA STI Report Series, which includes the following report types:

- TECHNICAL PUBLICATION. Reports of completed research or a major significant phase of research that present the results of NASA programs and include extensive data or theoretical analysis. Includes compilations of significant scientific and technical data and information deemed to be of continuing reference value. NASA's counterpart of peerreviewed formal professional papers but has less stringent limitations on manuscript length and extent of graphic presentations.

- TECHNICAL MEMORANDUM. Scientific and technical findings that are preliminary or of specialized interest, e.g., quick release reports, working papers, and bibliographies that contain minimal annotation. Does not contain extensive analysis.

- CONTRACTOR REPORT. Scientific and technical findings by NASA-sponsored contractors and grantees.
- CONFERENCE PUBLICATION. Collected papers from scientific and technical conferences, symposia, seminars, or other meetings sponsored or cosponsored by NASA.

- SPECIAL PUBLICATION. Scientific, technical, or historical information from NASA programs, projects, and missions, often concerned with subjects having substantial public interest.

- TECHNICAL TRANSLATION. Englishlanguage translations of foreign scientific and technical material pertinent to NASA's mission.

Specialized services that complement the STI Program Office's diverse offerings include creating custom thesauri, building customized data bases, organizing and publishing research results ... even providing videos.

For more information about the NASA STI Program Office, see the following:

- Access the NASA STI Program Home Page at http://www.sti.nasa.gov

- E-mail your question via the Internet to help@sti.nasa.gov

- Fax your question to the NASA Access Help Desk at (301) 621-0134

- Telephone the NASA Access Help Desk at (301) 621-0390

- Write to:

NASA Access Help Desk

NASA Center for AeroSpace Information 7121 Standard Drive

Hanover, MD 21076 
NASA/TM-1999-209042

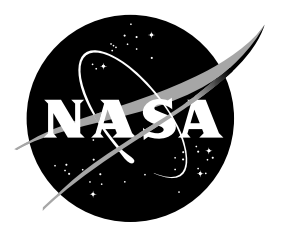

\section{Surface Segregation in Multicomponent Systems: Modeling of Surface Alloys and Alloy Surfaces}

Guillermo Bozzolo

Ohio Aerospace Institute, Cleveland, Ohio

John Ferrante

Cleveland State University, Cleveland, Ohio

Ronald D. Noebe, Brian Good, Frank S. Honecy, and Phillip Abel Glenn Research Center, Cleveland, Ohio

National Aeronautics and Space Administration

Glenn Research Center 


\section{Acknowledgments}

Fruitful discussions with N. Bozzolo are gratefully acknowledged. We also wish to thank C. Amador for his assistance in determining the first-principles parameters used.

This work was partially funded by the HITEMP and PPM programs at NASA Glenn Research Center, Cleveland, Ohio.

Available from

NASA Center for Aerospace Information 7121 Standard Drive

Hanover, MD 21076

Price Code: A04
National Technical Information Service 5285 Port Royal Road Springfield, VA 22100 Price Code: A04 


\title{
Surface Segregation in Multicomponent Systems: Modeling of Surface Alloys and Alloy Surfaces
}

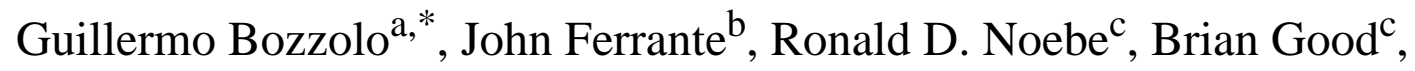 \\ Frank S. Honecy ${ }^{\mathrm{c}}$, Phillip Abel ${ }^{\mathrm{c}}$ \\ a Ohio Aerospace Institute, 22800 Cedar Point Road, Cleveland, Ohio 44142, USA \\ ${ }^{\mathrm{b}}$ Physics Department, Cleveland State Universtity, Cleveland, OH 44115, USA \\ ${ }^{\mathrm{c}}$ National Aeronautics and Space Administration, Glenn Research Center, Cleveland, $\mathrm{OH} 44135$,
}

\begin{abstract}
The study of surface segregation, although of great technological importance, has been largely restricted to experimental work due to limitations associated with theoretical methods. However, recent improvements in both first-principles and semi-empirical methods are opening the doors to an array of new possibilities for surface scientists. We apply one of these techniques, the BFS method for alloys, which is particularly suitable for complex systems, to several aspects of the computational modeling of surfaces and segregation, including alloy surface segregation, structure and composition of alloy surfaces, and the formation of surface alloys. We conclude with the study of complex NiAl-based binary, ternary and quaternary thin films (with $\mathrm{Ti}, \mathrm{Cr}$ and $\mathrm{Cu}$ additions to $\mathrm{NiAl}$ ). Differences and similarities between bulk and surface compositions are discussed, illustrated by the results of Monte Carlo simulations. For some binary and ternary cases, the theoretical predictions are compared to experimental results, highlighting the accuracy and value of this developing theoretical tool.
\end{abstract}

Keywords: Surface Segregation, Alloys, Surface Alloys, Monte Carlo Simulations, BFS Method, Intermetallics

*Corresponding author. Tel. +1 216433 5824; fax: +1 440962 3057;

Email: Guillermo.H.Bozzolo@grc.nasa.gov 


\section{Introduction}

Due to energetic factors, the composition and structure of the surface of metallic alloys usually vary from those of the bulk material. These differences between surface and bulk composition have been the subject of numerous theoretical and experimental studies, especially since a detailed knowledge of surface composition and structure is a prerequisite for understanding macroscopic surface properties such as adhesion and catalysis [1-4]. While there has been substantial

effort in examining surfaces of pure elements and the study of the surface behavior of binary alloys is rapidly developing, our knowledge of surface behavior rapidly deteriorates as alloys become more complex. In contrast to the abundant work done on binary systems, few studies of ternary or higher component systems have been performed [5].

Yet, a better understanding of alloy surface properties would be highly desirable and could even lead to advances in our ability to effectively design alloys for surface-related applications, especially if the appropriate design tools existed. When mature, computer-aided alloy design would be preferred to the traditional and often expensive empirical approach used for predicting bulk properties, where new alloys are developed from an incremental extrapolation of properties in a large database, generally by trial-and-error.

Computer methods can be divided into two broad categories: ab-initio methods and semiempirical approaches. However, both of these processes still have basic limitations associated with them. Ab-initio approaches, while deemed highly accurate and informative, involve computer intensive calculations, which place practical limits on the complexity of the systems that can be studied. In general, these methods are not efficient handling systems that contain three or more elemental constituents, changes in crystallographic structure, and dynamic processes such as annealing. These problems place practical but severe limitations based on computer time requirements. Therefore, ab-initio approaches have been used mainly for only the simplest systems and problems.

On the other hand, semiempirical methods, while much more computationally efficient, have in the past been plagued with their own set of problems. Initially, these included the inability to properly represent the appropriate interactions in many-component systems and to deal with crystal structures that are more complex than the simple bcc, fcc and hcp symmetries. Progress in resolving these deficiencies over the last decade has resulted in a rapid growth in the area of 
semiempirical techniques [7-9]. However, methods that exhibit a great deal of accuracy for bulk materials often fail to reproduce even some of the most basic properties of surfaces. Therefore, most of the semiempirical methods currently available do not perform very well in solving problems related to alloy surfaces or thin films. There is an additional problem, associated with parameterization, that limits the flexibility of most semiempirical techniques. In general, their field of applicability to monoatomic or very specific binary systems for which system-specific parameterization or potentials are used, severely limits the transferability of a developed model to even a similar problem without having to completely re-parameterize the process.

Consequently, our contention is that growth in theoretical surface analysis of higher order alloys has been frustratingly limited. But, with the proper tool, such a technique could provide a wealth of reliable informationin this area, as we will begin to demonstrate in this paper. A recently developed approximate technique introduced by Bozzolo, Ferrante and Smith (BFS) [7], shows great promise in efficiently dealing with complex systems and geometries, with the added advantage of a simple parameterization scheme. The BFS method is free of most of the limitations discussed above that are characteristic of other comparable semiempirical methods. Most importantly, it imposes no restrictions on the type or number of metallic elements or on the crystallographic structure under study.

The goal of this paper is to examine the contribution of BFS and other semiempirical methods to the study of surface properties. Although the emphasis is on surface segregation, it is our objective to provide a comprehensive description of the different issues relevant to surface segregation within the framework of a powerful computational technique. We therefore begin this presentation with a brief description of the BFS method, followed by a simple analysis of alloy surface energies and structure. Previous attempts to tackle this fundamental problem by means of other computationally efficient techniques with comparable physical basis have shown substantial limitations. Most techniques rely on the derivation of interaction potentials or energy functionals parameterized by bulk properties, which fail to handle such a drastic defect as a surface with similar accuracy. The BFS method, due to the concepts underlying its formulation, performs as well in surface analysis calculations as it does in the bulk, as we will show later, thus providing a solid foundation for the study of surface composition of higher order alloys.

We continue our presentation by investigating, both analytically and numerically, the process of surface segregation. In doing so, we exploit both the mathematical simplicity of BFS for the 
derivation of simple concepts for describing the segregation process as well as the computational efficiency of the BFS method by performing large scale Monte Carlo temperature dependent simulations for the determination of surface segregation profiles of binary alloys. We also discuss the related problem of surface alloying, with emphasis on the formation of surface alloys of immiscible metals.

The second half of this paper is devoted to a numerical analysis of ternary and quaternary alloy surfaces. Due to the complex nature of this problem, we first comment on the bulk properties of the alloys considered, as predicted by BFS and demonstrated experimentally. Having established the validity of the methodology and the accuracy of the parameterization used, we then perform an extensive analysis via large scale Metropolis Monte Carlo simulations of the surface composition of ternary and quaternary NiAl-based alloys which have yet to be examined experimentally.

\section{The BFS Method}

Since its inception a few years ago, the BFS method has been applied to a variety of problems, starting with the basic analysis of bulk properties of solid solutions of fcc and bec binary alloys (heat of formation [10], lattice parameter [11]) and more specific applications like the energetics of bimetallic tip-sample interactions in an atomic force microscope [12] as well as Monte Carlo simulations of the temperature dependence of surface segregation profiles in $\mathrm{Cu}-\mathrm{Ni}$ alloys [13]. The validity of these studies is largely based on a detailed analysis of the corresponding surface structure of the metallic alloys studied[14]. More recently, the ability of the BFS method to deal with alloy surfaces as well as the phase stability in bulk alloys has been illustrated in analytical studies and numerical simulations of surface alloys $[15,16]$ and the design of Ni-based quaternary alloys [17], providing a strong foundation for the work presented in this paper. An additional advantage of BFS is that it allows for deriving simple, approximate expressions which describe the trends in segregation as well as elucidating the driving mechanisms for these phenomena [18]. As a consequence of the ideas underlying the foundation of BFS, simple expressions for predicting the composition dependence of bulk alloy properties based solely on pure component proper-

ties have been recently derived (the BF rule) [19], providing an alternative to the commonly used 
Vegard's law [20]. In particular, the BF rule was used in the present work to compute the lattice parameter of the ternary and quaternary systems studied, for which no experimental data yet exist.

In what follows we present an operational review of the BFS method. Due to its novel way of partitioning the energy into structural and chemical contributions, this presentation should be complemented with a review of previous applications, in order to familiarize the reader with the main concepts discussed below [7,10-14,16-19].

The BFS method is based on the idea that the energy of formation $\Delta H$ of an arbitrary alloy structure is the superposition of individual contributions $\varepsilon_{i}$ of all the atoms in the alloy [7],

$$
\Delta H=\sum_{i} \varepsilon_{i}=\sum_{i}\left(e_{i}^{\prime}-e_{i}\right)
$$

where $e^{\prime}{ }_{i}$ is the energy of atom $i$ in the alloy and $e_{i}$ is the energy of this atom in its equilibrium single crystal. Each individual contribution $\varepsilon_{i}$ can be interpreted as the superposition of two distinct processes that, properly coupled, attempt to simulate the process of alloy formation: a strain energy, $e_{i}^{S}$, and a chemical energy, $\quad \varepsilon_{i}^{c h e m}=e_{i}^{C}-e_{i}^{C_{0}}$, linked by a coupling function $g_{i}$

$$
\varepsilon_{i}=e_{i}^{S}+g_{i}\left(e_{i}^{C}-e_{i}^{C_{0}}\right)
$$

where $i$ denotes the atomic species of a given atom ( $e^{C_{0}}$ is a reference energy to be defined later). The strain energy, $e_{i}^{S}$, accounts for the actual geometrical distribution of the atoms surrounding atom $i$, computed as if all its neighbors were of the same atomic species as atom $i . e_{i}^{S}$ is then evaluated with any suitable technique for computing the energy of a monoatomic crystal. In this work, as in all previous applications of BFS, we use Equivalent Crystal Theory (ECT) [21]. ECT provides a simple, straightforward way to compute the strain energy in a framework consistent with the formulation of BFS for computing the chemical energy.

Based on the assumption that the universal binding energy relationship of Rose et al. [22] contains all the relevant information concerning a given single-component system, we write

$$
e_{i}^{S}=E_{C}^{i} F\left(a_{i}^{S^{*}}\right)
$$

where $E_{C}^{i}$ is the cohesive energy of element $i$ and where 


$$
F\left(a^{*}\right)=1-\left(1+a^{*}\right) e^{-a^{*}}
$$

where $a_{i}^{S^{*}}$, given by

$$
a_{i}^{S^{*}}=q \frac{\left(a_{i}^{S}-a_{e}^{i}\right)}{l_{i}}
$$

is a scaled lattice parameter which contains the structural information of the defect crystal, in that its magnitude and sign indicate a measure of the defect. The quantities $a_{e}^{i}$ and $l$ are the equilibrium lattice parameter and scaling length, respectively, of a pure defect-free crystal of species $i$. The factor $q$ in Eq. 5 is the ratio between the equilibrium Wigner-Seitz radius and the equilibrium lattice parameter for element $i$. Within the framework of ECT, $a_{i}^{S}$ can be interpreted as the lattice parameter of an ideal, perfect crystal, i.e. the equivalent crystal, uniformly expanded or contracted so that the energy per atom is the same as the energy of atom $i$ in the actual, defect crystal.

The coupling term, $g_{i}$, is related to the strain energy in the sense that it contains information on the structural defect included in $e_{i}^{S}$. In evaluating $e_{i}^{S}$, the scaled lattice parameter $a_{i}^{S^{*}}$ can be easily obtained from Eq. 5, from which the coupling term $g_{i}$ becomes

$$
g_{i}=e^{-a_{i}^{S^{*}}}
$$

Using ECT for computing the strain energy introduces the added advantage that $a_{i}^{S}$ (and thus $a_{i}^{S^{*}}$ ) is directly obtained by solving the ECT equation for the defect crystal, as shown below.

In general, the ECT equation for computing the strain energy reads

$$
N R_{1}^{p} e^{-\alpha R_{1}}+M R_{2}^{p} e^{-\left(\alpha+\frac{1}{\lambda}\right) R_{2}}=\sum_{j} r_{j}^{p} e^{-\left(\alpha+S\left(r_{j}\right)\right) r_{j}}
$$

(see Ref. 7 for details) where $N(M)$ is the number of (next-) nearest-neighbors in the equivalent crystal of species $i$ and $R_{1}\left(R_{2}\right)$ is the (next-) nearest-neighbor distance (to be determined from Eq. 7) in the equivalent crystal of lattice parameter $a_{i}^{S}$ and where the quantities $p, \alpha, \lambda$, and the screening function $S$ are as defined in Ref. 21. See Table 1 for example values. In this formulation, the parameter $\alpha$ plays a key role as it parameterizes the electron density in the overlap region between to neighboring atoms. The sum on the right hand side of Eq. 7 runs over all nearest- and 
next-nearest-neighbors $j$ of atom $i$ at a known distance $r_{j}$ from the reference atom. Eq. 7 is then solved for the lattice parameter of the equivalent crystal $a_{i}^{S}$ via $R_{1}$ and $R_{2}$, which are the corresponding nearest- and next-nearest-neighbor distances in the equivalent crystal. The strain energy is then computed with Eq. 3.

For the particular case where all the neighboring atoms are located at lattice sites, $r_{j}=r_{1}$ and $S\left(r_{1}\right)=0$ for nearest-neighbors; $r_{j}=r_{2}$ and $S\left(r_{2}\right)=1 / \lambda$ for next-nearest-neighbors; and if $n$ is the actual number of nearest-neighbors and $m$ is the corresponding number of next-nearest-neighbors in the real crystal, then Eq. 7 is simply

$$
N R_{1}^{p} e^{-\alpha R_{1}}+M R_{2}^{p} e^{-\left(\alpha+\frac{1}{\lambda}\right) R_{2}}=n r_{1}^{p} e^{-\alpha r_{1}}+m r_{2}^{p} e^{-\left(\alpha+\frac{1}{\lambda}\right) r_{2}}
$$

Rigorously, the computation of the strain energy includes four terms (see Ref. 21). In this work, we neglect the three- and four-body terms dealing with the bond angle and face-diagonal anisotropies and retain only the two-body term that accounts for bond-length anisotropies [7,21], which we expect to be relevant for atoms in the top (surface) layers. The higher order terms would be proportional to the small local fluctuations of the atomic positions around the equilibrium lattice sites. We expect that the leading term (Eq. 8), will adequately account for these small distortions.

In the chemical contribution $e_{i}^{C}$, as opposed to the strain energy term, the surrounding atoms retain their chemical identity, but are forced to be in equilibrium lattice sites. The changes in electron density in the overlap region between neighboring atoms is now different from that used in the calculation of the strain energy (where all atoms are of the same atomic species). These changes are taken into account by introducing a small perturbation to the parameter $\alpha$ of the reference atom, denoting the fact that a neighboring atom could be of a different element. We therefore introduce the BFS parameters $\Delta_{k i}\left(\Delta_{i k}\right)$ to denote the influence of a neighbor of species $k(i)$ on the electron density in the vicinity of the reference atom $i(k)$, so that $\alpha_{i k}=\alpha_{i}+\Delta_{k i}$ $\left(\alpha_{k i}=\alpha_{k}+\Delta_{i k}\right)$ (see Table 2) is used for the interaction between atoms $i$ and $k$. If $N_{i k}\left(M_{i k}\right)$ denotes the number of species $k$ (next) nearest-neighbors of the atom in question (of species $i$ ), then the BFS equation to be solved in order to find the equivalent lattice parameter $a_{i}^{C}$ is 


$$
N R_{1}^{p_{i}} e^{-\alpha_{i} R_{1}}+M R_{2}^{p_{i}} e^{-\left(\alpha_{i}+\frac{1}{\lambda_{i}}\right) R_{2}}=\sum_{k} N_{i k} r_{1_{i}}^{p_{i}} e^{-\alpha_{i k} r_{1_{i}}}+\sum_{k} M_{i k} r_{2_{i}}^{p_{i}} e^{-\left(\alpha_{i k}+\frac{1}{\lambda_{i}}\right) r_{2_{i}}}
$$

where $N(M)$ is the number of (next) nearest-neighbors in the equivalent crystal of species $i$. $R_{1}\left(R_{2}\right)$ is the (next) nearest-neighbor distance in the equivalent crystal of lattice parameter $a_{i}^{C}$. The distances $r_{1}$ and $r_{2}$ are the equilibrium nearest- and next-nearest-neighbor distances in an equilibrium crystal of species $i$, respectively. The chemical energy is then computed with

$$
e_{i}^{C}=\gamma E_{C}^{i} F\left(a_{i}^{C^{*}}\right)
$$

\begin{tabular}{|c|c|c|c|c|c|c|c|}
\hline \multicolumn{4}{|c|}{ Experimental values } & \multicolumn{4}{|c|}{ ECT parameters } \\
\hline Atom & $\begin{array}{c}\text { Lattice } \\
\text { Parameter } \\
\text { (A) }\end{array}$ & $\begin{array}{c}\text { Cohesive } \\
\text { Energy } \\
(\mathrm{eV})\end{array}$ & $\begin{array}{l}\text { Bulk } \\
\text { Modulus } \\
(\mathrm{GPa})\end{array}$ & $\mathrm{p}$ & $\begin{array}{c}\alpha \\
\left(\AA^{-1}\right)\end{array}$ & $\begin{array}{c}\lambda \\
\left(\AA^{-1}\right)\end{array}$ & $\begin{array}{l}1 \\
(\stackrel{\circ}{\AA})\end{array}$ \\
\hline $\mathrm{Ni}$ & 3.524 & 4.435 & 187.6 & 6 & 3.015 & 0.759 & 0.270 \\
\hline $\mathrm{Al}$ & 4.05 & 3.34 & 79.4 & 4 & 2.105 & 0.944 & 0.336 \\
\hline $\mathrm{Pd}$ & 3.89 & 3.94 & 195.4 & 8 & 3.612 & 0.666 & 0.237 \\
\hline $\mathrm{Ag}$ & 4.086 & 2.96 & 108.7 & 8 & 3.337 & 0.756 & 0.269 \\
\hline $\mathrm{Cu}$ & 3.615 & 3.50 & 142.0 & 6 & 2.935 & 0.765 & 0.272 \\
\hline $\mathrm{Au}$ & 4.078 & 3.78 & 180.3 & 10 & 4.339 & 0.663 & 0.236 \\
\hline $\mathrm{Pt}$ & 3.92 & 5.85 & 288.4 & 10 & 4.535 & 0.666 & 0.237 \\
\hline
\end{tabular}

Table 1. Experimental values [21] for the lattice parameter, cohesive energy and bulk modulus for the fcc elements used in this work. The last four columns display the resulting Equivalent Crystal Theory (ECT) [21] parameters determined from fitting to the experimental values of the heat of solution. $p$ is related to the principal quantum number $n$ for the atomic species considered $(p=2 n-2), \alpha$ parameterizes the electron density in the overlap region between two neighboring atoms, $\lambda$ is a screening factor for atoms at greater than nearest-neighbor distances and $l$ is a scaling length needed to fit the lattice parameter dependence of the energy of formation with the universal binding energy relationship of Rose et al. (22). 
and

$$
e_{i}^{C_{o}}=\gamma_{0} E_{C}^{i} F\left(a_{i}^{C_{0}^{*}}\right)
$$

where $\gamma\left(\gamma_{0}\right)=1$ if $a_{i}^{C^{*}}\left(a_{i}^{C_{0}^{*}}\right) \geq 0$ and $\gamma\left(\gamma_{0}\right)=-1$ otherwise, and

$$
a_{i}^{C^{*}}=q\left(a_{i}^{C}-a_{e}^{i}\right) / l_{i}
$$

The scaled lattice parameter $a_{i}^{C^{*}}$ is obtained from Eq. 9 with the parameters $\Delta_{i k}$ listed in Table 2, and $a_{i}^{C_{0}{ }^{*}}$ is computed by following the same procedure used for $a_{i}^{C^{*}}$ but with $\alpha_{i k}=\alpha_{i}$. As defined, $e_{i}^{C_{0}}$ plays the role of a reference chemical energy, as it frees $\varepsilon^{C}$ from the influence of any structural defect.

When dealing with alloys with an underlying crystallographic structure similar to that of the constituents (i.e., $\mathrm{Ni}_{3} \mathrm{Al}$ is an fcc-based alloy, as well as its components $\mathrm{Ni}$ and $\mathrm{Al}$ ), the parameterization of the method can be easily done using readily available experimental data. Later in this work, however, we will explore situations where the alloy has a different crystallographic structure than that of its constituents (i.e., NiAl is a bcc-based alloy, with fcc elements $\mathrm{Ni}$ and $\mathrm{Al}$ ). The BFS method requires consistency between the crystallographic structure of the alloy under study and its constituents. The cohesive energy, lattice parameter and bulk modulus of $\mathrm{Ni}$ and $\mathrm{Al}$ (and any other alloying addition) in the bcc phase will thus be needed, for which, obviously, there is no experimental data available. Such a calculation can be easily carried out using first-principles methods. Moreover, simple first-principles approaches can be used in either case (whether experimental data is available or not), thus providing us with an efficient way of determining the necessary parameters regardless of the type of alloy or elements under consideration or the availability of experimental data. All current applications of BFS are based on this approach, where all parameters used are generated using first-principles methods, introducing consistency in the formulation and application of the method.

In this work, however, we will use both approaches (i.e., experimental and theoretical parameters) with the sole purpose of investigating the role of the parameterization scheme used in the final results. Therefore, the first few examples to be shown in later sections (surface energies, surface relaxation, surface segregation of binary systems and surface alloying) will be based on ECT 


\begin{tabular}{|l|l|l|l|l|l|l|l|}
\hline $\mathbf{j l i}$ & $\mathbf{N i}$ & $\mathbf{A l}$ & $\mathbf{C u}$ & $\mathbf{A g}$ & $\mathbf{A u}$ & $\mathbf{P d}$ & $\mathbf{P t}$ \\
\hline $\mathrm{Ni}$ & & -0.0861 & 0.0309 & & -0.0622 & -0.0396 & -0.0609 \\
\hline $\mathrm{Al}$ & -0.0657 & & -0.0526 & -0.0499 & -0.0501 & & \\
\hline $\mathrm{Cu}$ & -0.0163 & -0.0626 & & -0.0308 & -0.0604 & -0.0495 & -0.0585 \\
\hline $\mathrm{Ag}$ & & 0.0475 & -0.0391 & & -0.0333 & -0.0451 & \\
\hline $\mathrm{Au}$ & -0.0506 & -0.0853 & -0.0513 & -0.0227 & & -0.0460 & \\
\hline $\mathrm{Pd}$ & -0.0478 & & -0.0495 & -0.0178 & -0.0345 & & \\
\hline $\mathrm{Pt}$ & -0.0537 & & -0.0441 & & & & \\
\hline
\end{tabular}

Table 2: BFS parameters $\Delta_{i j}$ and $\Delta_{j i}$ (see text).

and BFS parameters determined experimentally for the fcc elements used (see Tables 1 and 2). In the second half of this paper, we will explore bcc-based systems with a mixture of bcc, fcc and hcp constituents, highlighting the need of a reliable source of parameters when experimental data is not available.

Table 1 lists the pure element ECT parameters for the fcc elements used in the first few applications. Table 2 lists the corresponding BFS parameters for A-B interactions $\left(\Delta_{\mathrm{AB}}\right.$ and $\left.\Delta_{\mathrm{BA}}\right)$ obtained by fitting to the experimental values of the heat of solution in the dilute limit.

We should emphasize that in the context of BFS, the strain and chemical energy contributions differ substantially in meaning from the ones these terms have in other approaches. The BFS strain energy is related to the usual strain only in that the atomic locations are those found in the actual alloy: the BFS strain energy of a given atom is then the actual strain that it would have in a monoatomic crystal of the same species as the reference atom. Likewise, the BFS chemical contribution is related to the usual chemical energy in that the actual chemical composition of the alloy is taken into account, but with the neighboring atoms located in ideal atomic sites: the BFS chemical energy of a given atom is then the actual chemical energy in an ordered environment with the lattice spacing characteristic of the equilibrium lattice of the reference atom. Therefore, the BFS contributions are, in a sense, a particular combination of the actual strain and chemical energies. We refer the reader to previous applications of BFS for more insight on this issue [7,10-14,16-19], and the success of this particular formulation. 


\section{Surface Energy and Structure}

As discussed earlier, the purpose of this work is to test the ability of approximate techniques to describe the basic mechanisms that are necessary to provide a complete description of alloy surface structure and composition. In what follows, we will address each one of these issues separately.

\section{3.a Surface Energy}

In this section we concentrate on the determination of surface energies, multilayer relaxation and individual atomic displacements off the surface plane for mixed composition truncations of ordered binary alloys. The minimization of the surface energy for the many possible alloy surface configurations determines the nature of surface segregation. Multilayer relaxations involve at best exceedingly small changes in position, and comparable changes in surface energy, whose minimization determines the final geometry of the surface. In order for a semiempirical method to be useful, it must accurately account for such small energy differences. The calculation of surface energies and multilayer relaxations using BFS is a straightforward and computationally economical procedure. Ref. 7 provides the working equations necessary for that task. More importantly, simple approximations can be made that allow for an easy interpretation of the qualitative behavior of the system under study.

The success of BFS in reproducing experimentally observed structural surface properties relies in the ability of ECT (used in the calculation of the BFS strain energy) to properly describe such an extended defect as a surface [21].

Table 3 compares some experimental results for surface energies of polycrystalline surfaces of $\mathrm{Ni}, \mathrm{Au}$ and $\mathrm{Cu}$ with BFS (see Ref. 23 for a comprehensive presentation of such results) and the Embedded Atom Method (EAM) [8]. Unfortunately, there are a limited number of experimental results available for surface energies and they are generally limited to polycrystalline surfaces because of the difficulty in performing the experiments. For this reason we present an average of values for the (111), (100) and (110) planes, the lowest energy planes for both BFS and EAM results, in addition to the calculated surface energies for each plane. As can be seen in Table 3, the BFS results agree quite well with experiment ( 10\%) and EAM [24] results tend to underestimate 


\begin{tabular}{|l|l|l|l|}
\hline Method & Au & Cu & Ni \\
\hline Experiment & $1500-1560$ & $1770-2016$ & $2240-2664$ \\
\hline BFS & $1132 / 1583 / 1619(1445)$ & $1767 / 2309 / 2373(2150)$ & $2274 / 2982 / 3073 \quad(2776)$ \\
\hline EAM & $790 / 918 / 980 \quad(896)$ & $1170 / 1280 / 1400(1283)$ & $1450 / 1580 / 1730(1587)$ \\
\hline
\end{tabular}

Table 3: Surface energies (in erg/ $\mathrm{cm}^{2}$ ) of monoatomic crystals of $\mathrm{Au}, \mathrm{Cu}$ and Ni. The first row indicates the range of experimental values, the second and third rows indicate the BFS and EAM [8] results, respectively, for the (111), (100) and (110) surfaces, with their average values between parenthesis.

\begin{tabular}{|l|l|l|l|l|l|}
\hline Method & $\mathbf{( 1 0 0 ) 1 : 1}$ & $\mathbf{( 1 0 0 ) 1 : 0}$ & $\mathbf{( 1 1 0 ) 1 : 1}$ & $\mathbf{( 1 1 0 ) 1 : 0}$ & $\mathbf{( 1 1 1 ) 3 : 1}$ \\
\hline BFS $[14]$ & 2852 & 3168 & 3117 & 3964 & 2411 \\
\hline EAM $[24]$ & 1620 & 1885 & 1730 & 1920 & 1645 \\
\hline
\end{tabular}

Table 4: Surface energies (in erg/ $/ \mathrm{cm}^{2}$ ) for different truncations of $\mathrm{Ni}_{3} \mathrm{Al}$, labeled by the truncation plane and ratio of $\mathrm{Ni}$ to $\mathrm{Al}$ atoms in the surface.

( $40 \%)$. The relative failure of EAM to reproduce the observed experimental values can be traced back to the definition of the embedding function, the main element in its formalism. This function is determined under the assumption that any given atom is in a bulk environment, which is clearly not the case in the vicinity of a surface.

Table 4 displays the corresponding predictions for $\mathrm{Ni}_{3} \mathrm{Al}$ surfaces by EAM [24] and BFS [14]. The BFS predictions are supported by the results in Table 3, which raise the necessary confidence for further predictions (multilayer relaxation) based on the behavior of the surface energy as a function of interatomic distance.

\section{3.b Surface Relaxation}

Much effort has been devoted to the study of surface relaxation in metals and, to a lesser extent, alloys. Several recent experiments have provided insights into the phenomenon of surface relaxation and composition in the case of alloys and correspondingly a few theoretical studies 


\begin{tabular}{|c|c|c|c|c|c|c|c|}
\hline & \multirow[b]{2}{*}{ Face } & \multirow{2}{*}{$\begin{array}{l}\text { Surface } \\
\text { energy } \\
\left(\mathrm{erg} / \mathrm{cm}^{2}\right)\end{array}$} & \multicolumn{4}{|c|}{$\begin{array}{l}\text { Change in interplanar spacing between layers } i \\
\text { and } j \text { for an atom } \mathrm{X} \text { in layer } i(\%)\end{array}$} & \multirow[b]{2}{*}{ Ripple } \\
\hline & & & $\Delta_{12}^{A}$ & $\Delta_{12}^{B}$ & $\Delta_{23}^{A}$ & $\Delta_{23}^{B}$ & \\
\hline$\overline{\mathrm{Al}-\mathrm{Ni}}$ & $\begin{array}{l}(100) 1: 1 \\
(100) 1: 0 \\
(100) 0: 1 \\
(110) 1: 0 \\
(110) 0: 1\end{array}$ & $\begin{array}{l}2028.52 \\
2887.57 \\
788.52 \\
3156.69 \\
3987.35 \\
3169.63 \\
2074.33 \\
3885.99 \\
3296.91 \\
2575.54\end{array}$ & $\begin{array}{l}+3.92(0.82) \\
-5.46(0.48) \\
-3.73(0.28) \\
-0.54(0.49) \\
-7.17(1.24) \\
-2.80(2.02) \\
-7.98(0.79) \\
+5.08(0.39)\end{array}$ & $\begin{array}{l}-11.14(0.54) \\
+13.05(1.18)\end{array}$ & $\begin{array}{l}+0.94(0.64) \\
+1.30(0.45) \\
+5.94(0.56) \\
+0.98(0.96) \\
+0.29(2.34) \\
+3.08(1.92) \\
-1.54(1.39) \\
+11.07(0.38)\end{array}$ & $\begin{array}{l}+6.56(0.25) \\
+0.00(0.00) \\
+2.59(0.29) \\
+0.66(1.14) \\
\\
-10.46(0.7) \\
-1.40(2.16) \\
+2.34(1.13)\end{array}$ & $\begin{array}{l}0.05310(0.0445) \\
0.16545(0.0327)\end{array}$ \\
\hline$\overline{\mathrm{Cu}-\mathrm{Au}}$ & $\begin{array}{l}(100) 1: 1 \\
(100) 1: 0 \\
(100) 0: 1 \\
(110) 1: 0 \\
(110) 0: 1\end{array}$ & $\begin{array}{l}1451.49 \\
1922.98 \\
2572.39 \\
2205.44 \\
1344.69 \\
2453.12 \\
1999.26 \\
2649.79 \\
2033.18 \\
1309.49\end{array}$ & $\begin{array}{l}-1.83(0.18) \\
-6.51(0.30) \\
-0.75(0.93) \\
+2.36(0.33) \\
-3.63(0.85) \\
-11.63(0.4) \\
-8.07(0.61) \\
+8.60(0.22)\end{array}$ & $\begin{array}{l}-1.66(1.25) \\
+16.68(0.52)\end{array}$ & $\begin{array}{l}+8.87(0.18) \\
+1.67(0.28) \\
+0.20(0.36) \\
+10.47(0.35) \\
+10.00(0.85) \\
-8.79(0.96) \\
+7.56(1.17) \\
+15.93(0.20)\end{array}$ & $\begin{array}{l}-2.99(0.61) \\
-1.65(0.86) \\
+2.48(0.87)\end{array}$ & $\begin{array}{l}0.15423(0.0131) \\
0.14941(0.0182)\end{array}$ \\
\hline $\mathrm{Ni}-\mathrm{Pt}$ & $\begin{array}{l}(100) 1: 1 \\
(100) 1: 0 \\
(100) 0: 1 \\
(110) 1: 0 \\
(110) 0: 1\end{array}$ & $\begin{array}{l}2153.11 \\
2577.37 \\
3248.61 \\
2759.94 \\
2228.15 \\
3355.64 \\
2519.11 \\
3234.27 \\
3057.54 \\
2042.79\end{array}$ & $\begin{array}{l}-4.63(0.18) \\
-9.03(0.29) \\
-3.75(0.68) \\
-0.46(0.29) \\
-4.79(0.77) \\
-11.94(0.4) \\
-9.83(0.48) \\
+3.67(0.20)\end{array}$ & $\begin{array}{l}-2.66(1.13) \\
+11.18(0.49)\end{array}$ & $\begin{array}{l}+5.87(0.19) \\
-0.66(0.26) \\
+7.99(0.30) \\
-1.94(0.33) \\
+8.72(1.08) \\
+5.24(1.25) \\
-9.56(0.83) \\
+11.22(0.19)\end{array}$ & $\begin{array}{l}-3.38(0.61) \\
+6.98(1.14) \\
+2.84(0.17)\end{array}$ & $\begin{array}{l}0.10835(0.0274) \\
0.16040(0.0148)\end{array}$ \\
\hline
\end{tabular}

Table 5: Surface energies and planar relaxation of the two top layers of several low-index faces of the $\mathrm{L1}_{0}$ (first line for each surface truncation) and $\mathrm{L1}_{2}$ (second line) ordered structures. The number in parenthesis is a measure of the uncertainty in the theoretical prediction due to uncertainties in the input parameters used. The last column indicates the distance (in $\stackrel{\circ}{\text { ) }}$ between atoms of different species in the same (top) plane [14]. 
have shown good general agreement with experimental results. The first experiment that provided detailed information on the atomic positions of surface atoms in a truncated ordered alloy was the low energy electron diffraction (LEED) intensity analysis of a $\mathrm{NiAl}(110)$ surface by Davis and Noonan [25], finding strong evidence for a rippled surface, where the Al sites of the top layer (in the mixed-composition truncation) are displaced above the Ni sites by approximately $0.22 \mathrm{~A}$. This result was followed by EAM calculations by Chen et al. [26] confirming the main experimental features. Several theoretical and experimental studies were later performed to investigate similar phenomena in other ordered alloys $\left(\mathrm{Ni}_{3} \mathrm{Al}\right.$ [24-27], $\mathrm{Cu}-\mathrm{Au}$ [28-31], Pt-Ni [32]). In what follows, we present a brief overview of BFS predictions for these and other systems.

Table 5 shows BFS results for the surface energies [14], multilayer relaxations and, when applicable, the rippling of the topmost layer in a number of binary alloys with similar symmetry. With very few exceptions, most of these results are of a purely predictive nature, with the objective of encouraging future experiments along these lines.

Ref. 7 contains a comprehensive presentation of the results summarized in this paper. Moreover, as pointed out earlier, this work addresses the issue of the parameterization based on experimental data. Relying on such input can introduce a substantial degree of uncertainty in the results obtained with BFS or comparable methods that base their accuracy on the quality of the parameters used. These parameters are generally obtained from different experiments for different elements or alloys, thus introducing undesired inconsistency in the results. In addition, experimental values are often quoted with a margin of error that is difficult to translate into a corresponding 'error' in the theoretical predictions. Therefore, Table 5 introduces an 'error bar' for the BFS results (between parentheses), indicating the variation of the predicted values for planar relaxations due to changes of $1 \%$ in the input parameters. While in general this variation does not translate into substantial changes in the qualitative behavior of the predicted properties, it hints at the need to develop alternative parameterization schemes that would result in a greater consistency between different elements and alloys as well as a precise and unique value for predicted quantities. For this reason, as well as for the additional fact that some parameters are not available experimentally, we developed a new parameterization scheme - to be used in the second half of this paper- based on first-principles calculations, thus freeing BFS from any dependence on experimental input. 


\section{Surface Segregation and Surface Alloying}

\section{4.a Surface Segregation of Single Substitutional Impurities}

In keeping with the theme of this publication we present the results of applying BFS to surface segregation and surface alloying in binary alloys. We proceed by examining predictions of segregating species for dilute solutions in binary alloys [18], then examine predictions for surface concentration profiles of single crystal $\mathrm{Ni}-\mathrm{Cu}$ solutions [13], and finally present results of surface alloying for $\mathrm{Au}$ on a $\mathrm{Ni}(110)$ surface $[15,16]$. The qualitative and quantitative success of these predictions will, it is hoped, lend credence to the thin film, multi-component results to follow in the final section of this paper.

The heat of segregation is defined as the difference between the heat of formation of a semiinfinite A crystal with an impurity B located at a lattice site on plane $p$ ( $p=0$ is the surface plane) parallel to the surface, and the same structure but with the atom B located in a lattice site deep within the bulk $(p=b)$. In this calculation we ignore relaxation effects around the impurity (i.e., small atomic displacements of the atoms from their equilibrium positions) as well as surface relaxation of the host crystal in order to concentrate on the role of other factors as driving forces for segregation.

Consider a cell containing the impurity atom such that the host atoms on the boundary of this cell are insensitive to the presence of the impurity. The cell is a sufficiently large piece of the crystal which includes not only the impurity atom in layer $p$ and its surrounding neighbors, but also several other atoms located in intermediate layers $q(q=0,1, \ldots, p, \ldots, b)$. With this definition of the size of the cell we expect to properly take into account the presence of the free surface. The definition of the cell will depend on the particular crystal structure and face studied. For example, in the absence of planar relaxations in an fcc (100) face, all the atoms in the top two layers $(p=0,1)$ are affected by the presence of the surface (i.e., an atom in layer $\mathrm{p}=2$ has all its nearest and next nearest neighbors between layers $p=0$ and $p=4$ ). In the non-relaxed case, the intermediate layers between the surface region and the bulk do not contribute to the calculation of the heat of segregation, making the calculation easier, as it significantly reduces the number of atoms to be considered (a more complete discussion can be found in Ref. 18). Therefore, the nature of the 


\begin{tabular}{|c|c|c|c|c|c|c|}
\hline Host & Impurity & Exp. & BFS & $\Delta S_{S}^{(0)}$ & $e_{B_{0}}^{S}-e_{B_{b}}^{S}$ & $e_{B_{0}}^{S}-e_{A_{0}}^{S}$ \\
\hline $\mathrm{Cu}$ & $\mathrm{Ni}$ & + & + & + & + & + \\
\hline $\mathrm{Ni}$ & $\mathrm{Cu}$ & - & - & - & + & - \\
\hline $\mathrm{Ag}$ & $\mathrm{Cu}$ & + & + & + & + & + \\
\hline $\mathrm{Cu}$ & $\mathrm{Ag}$ & - & - & - & + & - \\
\hline $\mathrm{Ag}$ & $\mathrm{Pd}$ & & + & + & + & + \\
\hline $\mathrm{Pd}$ & $\mathrm{Ag}$ & - & - & - & + & - \\
\hline $\mathrm{Au}$ & $\mathrm{Cu}$ & + & + & + & + & + \\
\hline $\mathrm{Cu}$ & $\mathrm{Au}$ & - & - & - & - & - \\
\hline $\mathrm{Au}$ & $\mathrm{Ni}$ & + & + & + & + & + \\
\hline $\mathrm{Ni}$ & $\mathrm{Au}$ & - & - & - & - & - \\
\hline $\mathrm{Au}$ & $\mathrm{Pd}$ & + & + & + & + & + \\
\hline $\mathrm{Pd}$ & $\mathrm{Au}$ & - & - & - & + & - \\
\hline $\mathrm{Cu}$ & $\mathrm{Pd}$ & & - & - & + & - \\
\hline $\mathrm{Pd}$ & $\mathrm{Cu}$ & & + & + & + & + \\
\hline $\mathrm{Cu}$ & $\mathrm{Pt}$ & & - & - & - & - \\
\hline $\mathrm{Pt}$ & $\mathrm{Cu}$ & - & + & + & + & + \\
\hline $\mathrm{Ag}$ & $\mathrm{Au}$ & + & + & - & + & - \\
\hline $\mathrm{Au}$ & $\mathrm{Ag}$ & $?$ & + & + & + & + \\
\hline $\mathrm{Ni}$ & $\mathrm{Pd}$ & - & - & - & - & - \\
\hline $\mathrm{Pd}$ & $\mathrm{Ni}$ & + & + & + & + & + \\
\hline $\mathrm{Ni}$ & $\mathrm{Pt}$ & & - & - & - & - \\
\hline $\mathrm{Pt}$ & $\mathrm{Ni}$ & + & + & + & + & + \\
\hline
\end{tabular}

Table 6 : Segregation trends where (-) means segregation of the impurity occurs and (+) means it does not. (?) means that experimental results are ambiguous [18]. See text for an explanation of the quantities listed in the last three columns.

problem allows for a number of simplifications that result in a rather simple set of final working equations. We define the heat of segregation as,

$$
\Delta E_{S}^{(p)}=\Delta E_{[p, B]}-\Delta E_{[p, A]}
$$

where $\Delta E_{[p, B]}$ and $\Delta E_{[p, A]}$ are the energies of formation of the computational slab with a $B$ atom in layer $p$ and an $A$ atom in the same place, respectively. Using Eqs. 1 and 2, a somewhat straightforward derivation provides an explicit expression for $\Delta E_{S}{ }^{(p)}$ in terms of individual atomic contributions as well as the distinction between BFS strain and chemical energies, 


$$
\Delta E_{S}{ }^{(p)}=e_{B_{p}}^{S}-e_{B_{b}}^{S}-e_{A_{p}}^{S}-N e_{A_{b}}^{C_{1}}-M e_{A_{b}}^{C_{2}}+e^{-a_{B_{p}}^{S^{*}}} e_{B_{p}}^{C}+e^{-a_{B_{b}}^{S *}} e_{B_{b}}^{C}+\sum_{q} e^{-a_{A_{q}}^{S^{*}}}\left[n_{q}^{p} e_{A_{q}}^{C_{1}}+m_{q}^{p} e_{A_{q}}^{C_{2}}\right]
$$

where $e_{X_{i}}^{Y}$ is the strain $(Y=S)$ or chemical $(Y=C)$ energy of an atom of species $X(X=A, B)$ in plane $i$. $N$ and $M$ denote the number of nearest and next nearest neighbors of the atom in the bulk ( $N=12$ and $M=6$ for fcc elements) and $n_{q}^{p}\left(m_{q}^{p}\right)$ denotes the number of (next-) nearest-neighbors in layer $q$ of an atom located in layer $p$. The scaled lattice parameter $a_{X_{i}}^{Y^{*}}$ is obtained from Eq. 5. The evaluation of the different terms in Eq. 14 is a simple numerical exercise, which is even amenable to approximations that allow for the derivation of simple expressions for $\Delta E_{S}{ }^{(p)}$.

In Table 6 we present a summary of the segregating species comparing BFS predictions (Eq. 14) with experiment. We can see that there is excellent agreement in all the cases considered, with disagreement for only one out of the sixteen systems where experimental data was available, $\mathrm{Cu}$ in Pt. The heat of segregation predicted by BFS in this case is very small and may require a more careful consideration of the sensitivity to fitting parameters, and as well may be a situation where relaxation effects (due to the large mismatch between $\mathrm{Cu}$ and $\mathrm{Pt}$ ) cannot be ignored.

It is interesting to examine Eq. 14 in some detail as it provides a simple, analytical way to isolate the different mechanisms involved in segregation and their influence on the heat of segregation. Eq.14 shows that two distinct contributions are responsible for segregation: a strain term,

$$
\Delta S_{S}^{(p)}=e_{B_{p}}^{S}-e_{B_{b}}^{S}-e_{A_{p}}^{S}
$$

and a chemical term

$$
\Delta C_{s}^{(p)}=\Delta E_{s}^{(p)}-\Delta S_{s}^{(p)}
$$

both defined in the context of BFS (meaning that "strain" denotes a somewhat different effect from the one commonly associated with that name).

One immediate conclusion regarding the driving mechanism is drawn from the predictions which are summarized in Table 6: the strain energy contribution consistently has the same sign as the whole quantity $\Delta E_{s}^{(p)}$ indicating that the segregation trends obtained from Eq. 14 are dictated by the relative value of the strain energy, as one would expect. Moreover, two different contributions can be selected from the different terms that make up the strain energy: $e_{B_{p}}^{S}-e_{B_{b}}^{S}$, directly related to the structural strain induced by the presence of the impurity, and $e_{B_{p}}^{S}-e_{A_{p}}^{S}$, which indi- 
rectly reflects the difference in surface energies of the two participating species (note that, as defined, these are not competing mechanisms). The first quantity $e_{B_{p}}^{S}-e_{B_{b}}^{S}$ does not follow the correct trend for the total BFS energy in all cases, although it tends to do so as the lattice mismatch between species A and B increases. We then conclude that only when the lattice mismatch is sufficiently large so as to make $\Delta S<0$ can this effect be taken as the one responsible for identifying the segregating species. The 'surface energy' term, $e_{B_{p}}^{S}-e_{A_{p}}^{S}$, consistently reproduces the same trends as $\Delta E_{s}$ as a whole, as well as trends in experimental results. These results are displayed in Table 6: the first column shows typical experimental predictions [18] for the sign of $\Delta E_{s}^{(0)}$. The second column displays the corresponding results obtained from Eq. 14 and the last three columns the signs of $\Delta S_{s}{ }^{(0)}, e_{B_{0}}^{S}-e_{B_{b}}^{S}$ and $e_{B_{0}}^{S}-e_{A_{0}}^{S}$. A negative sign in the first two columns indicates that the impurity atom segregates to the surface. We have also examined the effects of surface relaxation on the segregating species and found that although naturally the heats of segregation were changed, the trends were not substantially affected for the systems examined (Ref. 18).

\begin{tabular}{|c|c|c|c|c|c|c|c|c|}
\hline Host & Imp. & Face & Method & $\Delta E_{S}^{(0)}$ & $\Delta E_{S}^{(1)}$ & $\Delta E_{S}^{(2)}$ & $\Delta E_{S}^{(3)}$ & $\Delta E_{S}{ }^{(4)}$ \\
\hline \multirow[t]{6}{*}{$\mathrm{Ni}$} & \multirow[t]{6}{*}{$\overline{\mathrm{Cu}}$} & (100) & BFS & -0.40001 & -0.02376 & 0.00015 & 0 & 0 \\
\hline & & & EAM & -0.426 & 0.045 & 0.002 & 0 & 0 \\
\hline & & (110) & BFS & -0.51134 & -0.0946 & -0.03232 & -0.00698 & 0 \\
\hline & & & EAM & -0.538 & -0.029 & 0.039 & 0.002 & 0 \\
\hline & & (111) & BFS & -0.31623 & -0.0217 & 0 & 0 & 0 \\
\hline & & & EAM & -0.304 & 0.029 & 0 & 0 & 0 \\
\hline \multirow[t]{6}{*}{$\mathrm{Cu}$} & \multirow[t]{6}{*}{$\mathrm{Ni}$} & (100) & BFS & 0.34956 & 0.03424 & 0.0003 & 0 & 0 \\
\hline & & & EAM & 0.233 & -0.147 & -0.002 & 0 & 0 \\
\hline & & (110) & BFS & 0.45169 & 0.1328 & 0.04786 & 0.01049 & 0 \\
\hline & & & EAM & 0.349 & -0.115 & -0.092 & -0.005 & 0 \\
\hline & & (111) & BFS & 0.29488 & 0.030725 & 0 & 0 & 0 \\
\hline & & & EAM & 0.160 & -0.090 & 0.002 & 0 & 0 \\
\hline
\end{tabular}

Table 7: Comparison of BFS and EAM results for the heat of segregation of a single substitutional impurity for different crystal faces ( $\Delta E_{S}{ }^{(n)}$ is the heat of segregation (in $\mathrm{eV}$ ) for the $n$th layer below the surface, the EAM results were taken from Ref. 33). 
As we move down to planes below the surface, one would expect the chemical effects to dominate as the influence of the surface is lessened, resulting in a more delicate balance between $\Delta S$ and $\Delta C$, which could translate into segregation patterns where certain layers are enriched and others are not, sometimes in an oscillatory fashion.

In studying the segregation trends for layers below the surface plane, the lack of reliable experimental data leads us to compare our predictions with other theoretical approaches. In Table 7, we compare BFS and EAM [33] results for the $\mathrm{Cu}-\mathrm{Ni}$ system, presenting the heats of segregation of a single substitutional impurity for different crystal faces. We can see that there is reason-

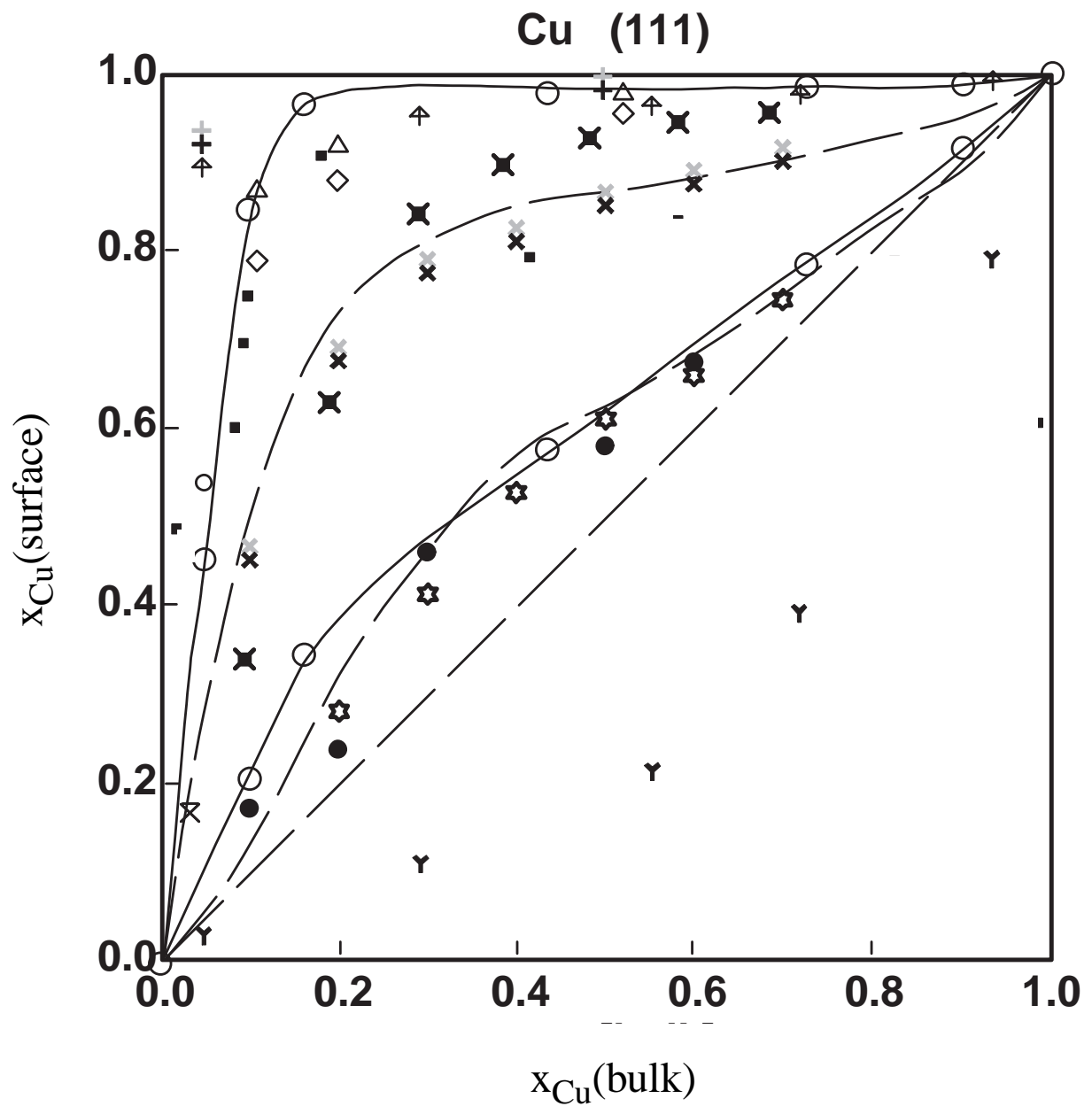

O BFS results $\uparrow$ EAM results

All other symbols: Experiment (Ref. 34-36)

Fig. 1: $\mathrm{Cu}(111)$ surface composition vs. $\mathrm{Cu}$ bulk composition: comparison between the experimental and theoretical results for the first and second planes. BFS results are indicated by circles, joined by a line to guide the eye. EAM results are indicated with $\hat{Y}$ for the top plane and $\mathbf{Y}$ for the second plane. All other symbols correspond to different experimental results. See Ref. 13 for a full description of experimental and theoretical results, as well as referenced experiments. 
able agreement for the top plane, but disagreement for the predictions of the lower lying planes. We will address this issue shortly, when we present a comparison of surface profiles with experiment.

\section{4.b. Segregation Profiles of Binary Alloys}

The generalization of the approach used in dealing with single impurities to the more general problem of determining segregation profiles [32] is beyond the scope of this paper. In dealing with this problem, we will concentrate on the results of computer simulations based on a Metropolis Monte Carlo algorithm using the BFS method at each step in the simulation.

We therefore conclude our discussion on surface segregation in binary alloys with the results from a segregation profile study of the (111) face of the $\mathrm{Cu}-\mathrm{Ni}$ system at $800 \mathrm{~K}$ for which there are substantial experimental and theoretical studies [33,34].

The theoretical procedure used to determine the segregation profiles is a variant of the Metropolis Monte Carlo method. Initially a computational cell consisting of 15 layers of 98 atoms is set up. A starting composition is chosen, and each atom within the cell is assigned a species probabilistically, so that the initial composition is uniform, both throughout the cell and within each individual layer. All but the last four layers are "active" in that their compositions are allowed to vary during the computation. For reasons having to do with the details of the energy calculations, the last four layers are static, and change neither composition nor the detailed distribution of the two chemical species within each layer. As the simulation proceeds, a pair of atoms of opposite species is chosen randomly from within the active region of the computational cell, and the total energy of the cell is computed using the BFS method. The chemical species of the two atoms are reversed and the total energy is recomputed. The reversal is accepted if it lowers the energy, or accepted with a probability $e^{-\Delta E / k T}$ if it raises the energy ( $\Delta E$ is the difference in energy between the initial and final state), according to the Metropolis criterion. The simulation is continued until the segregation profile attains a steady state.

Representative results of this simulation are presented in Fig. 1, where the $\mathrm{Cu}$ surface concentration is plotted as a function of bulk concentration. Even at low $\mathrm{Cu}$ concentration, there is a substantial increase of $\mathrm{Cu}$ in the surface region, consistent with the trend observed in Table 7. The BFS results are in excellent agreement with experiment for the whole composition range. We 
found that there is no concentration reversal for high $\mathrm{Cu}$ concentrations as found by Sakurai et al. [34]. Simulations were carried out as a function of temperature and crystal face. In all cases, monotonic profiles were found.

More importantly, we quantitatively predict $\mathrm{Cu}$ enrichment in the layer below the surface, in agreement with experiment but contrary to the depletion predicted by EAM [33] (Y's in Fig. 1). This disagreement between EAM and experiment can be seen already in Table 7, where Cu presence in the first layer below the surface is energetically unfavorable.

\section{4.c. Surface Alloys}

We conclude this section with a discussion of the BFS predictions of surface alloying in the $\mathrm{Au}$ on Ni system $[15,16]$. A discussion of other surface alloys can be found in Refs. 35 and 36. Although this is strictly speaking not surface segregation, the sensitivities needed to determine behavior are the same as for surface segregation.

The first demonstration of surface alloying of immiscible metals was performed with the scanning-tunneling microscopy studies of Nielsen et al. [15] for Au on Ni (110). A wealth of recent experiments $[35,36]$ has shown that the alloying patterns are unexpectedly complex, thus semiempirical techniques and the ensuing modeling efforts must predict, classify and understand these complexities.

Two different paths can be taken to understand this phenomenon from a theoretical standpoint: one, performing large scale numerical simulations and comparing the outcome with experimental results; another, performing detailed analytical calculations on a number of likely atomic distributions. While the first approach, computationally oriented, helps in the interpretation of the experimental results, the second approach results in a deeper physical insight into the inner workings of the process under study. For our study of surface alloying of immiscible metals, we chose the second approach, based on the development of a catalogue of configurations representing accessible states of the system, which may or may not describe the actual atomic distribution during the actual process of surface alloy formation. In doing so, we can obtain valuable information on the basic mechanisms guiding the process.

Zero-temperature BFS results are therefore based on an energy analysis of a large number of possible distributions of the adatoms. Starting with low coverage, a few adatoms are located either 
in specific sites in the overlayer or in substitutional sites in the surface plane with displaced substrate atoms. The energy of formation of these arrangements is computed similarly to the method just described for segregation and then the process of surface alloy formation (when the adatoms insert themselves in the substrate forming a one- or two-layer mix) is reconstructed. For this work, we used an approach where a set of possible configurations is determined, responding to the symmetries of the surface under study and concentrating on the type of atomic distributions that could have some physical meaning. Creating this type of 'catalogue' of possible or likely configurations is an alternative to a pure numerical analysis, with the advantage that one can extract from the results trends and features that cannot be obtained from am extensive numerical approach. While this form of 'static' analysis does not take into account thermal diffusion effects, it is to be expected that even within the constraints of the calculation (no planar relaxation or defect formation) the theoretical results should clearly indicate whether a configuration is favored or not.

Summarizing, a number of subtle effects which occur are correctly predicted by BFS. First, at low coverages, injection of a single atom is energetically favored in the surface plane of the $\mathrm{Ni}$, but not in the second layer. Second, insertion of $\mathrm{Au}$ dimers is favored in the surface layer over monomers, with again no insertion in the second layer. Third, Ni chains of the displaced Ni in the overlayer are formed along the close-packed direction. Finally, at higher Au coverage ( .5 monolayers), a phase separation occurs where the Au atoms form a homogeneous Au overlayer, reversing the process of surface alloy formation. Each of these features is predicted by BFS along with the quantitatively correct coverage for phase separation. Effective Medium Theory (EMT) explains these results theoretically [15] and presents a simple explanation which is also concluded from BFS for such a process. Moreover, the simple BFS formalism allows for the determination of the 'effective' coordination, i.e. the number of surface atoms that an adatom needs to be surrounded by, in order to reproduce its own bulk electronic environment. This concept, suggested in the original EMT analysis of this phenomenon [15] can easily be quantified and extended by means of the BFS equation actually providing a measure of effective coordination and its consequences [16]. For example, the presence of $\mathrm{Au}$ dimers in a Ni(110) surface plane is explained by the fact that the Au dimers find an electronic density similar to that found in a pure Au Crystal. This concept also explains why penetration to deeper layers is not favored and how specific ordering patterns can be achieved. 


\section{Surface composition of ternary and quaternary alloys}

Having shown the ability of the BFS method to deal with the different aspects of surface structure and composition of binary alloys, the main purpose of this section is to illustrate an application of the BFS method to surface studies of multicomponent metallic systems (beyond binary alloys) by showing theoretical results for films of binary, ternary and quaternary alloys of $\mathrm{Ni}, \mathrm{Al}$, $\mathrm{Cr}, \mathrm{Ti}$ and $\mathrm{Cu}$.

Our emphasis on NiAl-based alloys is due to the particular relevance they have in industrial applications [37] although, unfortunately, experimental data for thin films and surfaces of these systems is not currently available. The predictive nature of our results finds validation in the success of the BFS in reproducing the experimental evidence concerning the defect structure of Ni-Al [38], Ni-Al-Ti and Ni-Al-Cr [39] alloys. It is expected that the credibility raised on the BFS predictions based on the results shown in Refs. 38-39, together with the solid foundation built on previous BFS work on NiAl-based alloy structure, will translate into useful results in motivating and aiding the analysis of these complex systems, should experimental work be carried out in the future.

Except for a few comparative theoretical or experimental studies on the binary $\mathrm{Ni}-\mathrm{Al}$ system, there is virtually no other study - to the best of our knowledge- that deals with these multi-component systems, giving the results presented in this section a purely predictive character. Needless to say, the wide range of possibilities with such a large number of individual constituents demands a long, comprehensive study, which is impractical by any measure. Therefore, we limit this first attempt at analyzing high-order systems and their surface properties to two main issues: 1) a comparison of the surface composition with the bulk structure of the corresponding alloy and 2) a selection of Monte Carlo simulations for those systems for which some experimental background exists.

In this section we will study the behavior of bcc, fcc and hep elements in a bcc-based alloy. For consistency, we have to use parameters for the individual elements, as well as for the interactions between them, consistent with the underlying lattice of the alloy at hand. This requires the determination of pure element physical properties for which no experimental values exist, which have to be determined theoretically. 
In this work, we used the parameters $\Delta_{A B}$ and $\Delta_{B A}$ determined following the procedure outlined in Ref. 38. These parameters are obtained from first-principles, all-electron, density functional based calculations of the elemental constituents and ordered binary compounds of these elements. The particular implementation used in this work is the Linear-Muffin-Tin Orbitals (LMTO) method [40] in the Atomic Sphere Approximation. As mentioned above, in order to provide parameters to the BFS method, we need to calculate the equilibrium properties of the elemental solid for the same symmetry of the compound to be studied. This set of parameters is accurately described by the Local Density Approximation [41]. Thus, for this case, we have calculated $\mathrm{Ni}, \mathrm{Al}, \mathrm{Ti}, \mathrm{Cr}$ and $\mathrm{Cu}$ single element properties (lattice parameter, cohesive energy and bulk modulus) in the bcc phase, as well as the energy of formation and equilibrium lattice parameter of the B2 ordered NiAl, NiTi, NiCr, etc., phases, whether they exist in nature or not. Calculations were done for different values of the lattice parameter, and total energies were then fitted to the universal equation of state of Rose et al. [22].

The LMTO method uses a minimal basis set: in this work, we have used only $s, p$ and $d$ orbitals. All calculations were done with equivalent sampling of the Brillouin zone using, for the bcc lattice, $120 k$-points in the irreducible wedge. Apart from the parameters describing the equation of state of the element (lattice parameter, cohesive energy and bulk modulus), the parameterization of the BFS approach requires the formation energy of a single vacancy $\left(\mathrm{E}_{\mathrm{vac}}\right)$ in order to fix the ECT parameter $\alpha$. We have also calculated $\mathrm{E}_{\mathrm{vac}}$ with the LMTO method using a supercell approach. Studies of the convergence of this property as a function of the supercell size showed that, for the required precision in the calculation $(0.1 \mathrm{eV} /$ atom $)$ and within practical computational limits, a supercell of 8 atoms is necessary. As BFS is parameterized without considering the relaxation caused by the formation of a vacancy, no relaxation is allowed in the LMTO calculations.

The consistent parameterization of the BFS method also requires the calculation of the formation energy and equilibrium lattice parameter of an ordered binary compound. We have chosen the bcc based B2 structure for all the binary systems A-B (A, B = Ni, Al, Ti, Cr, Cu). This calculation for the compound is equivalent in the basis set and sampling of the Brillouin zone used for the pure elements.

The ECT and BFS parameters used in this work for $\mathrm{Ni}, \mathrm{Al}, \mathrm{Cr}, \mathrm{Ti}$ and $\mathrm{Cu}$ are listed in Tables 8 and 9, respectively. Once these parameters are computed, they remain the same for any calculation involving any of these elements, requiring no further adjustment or replacement. It should be 
noted that, within this parameterization scheme, the numerical values of the BFS parameters $\Delta_{A B}$ and $\Delta_{B A}$ can be easily obtained, as the simplicity of the BFS method allows for a simple analytical procedure for the determination of such parameters [42].

The validity of the parameters listed in Tables 8 and 9 relies on previous applications of BFS to the determination of the phase structure of bulk NiAl-based alloys with the same alloying additions considered in this work. Moreover, the same parameters can be used as long as either one of these elements appears in a bcc phase.

\begin{tabular}{|l|c|c|c|c|c|c|c|c|}
\hline \multicolumn{5}{|c|}{ LMTO results } & \multicolumn{3}{c|}{ ECT parameters } \\
\hline Atom & $\begin{array}{c}\text { Lattice } \\
\text { Parameter } \\
(\AA)\end{array}$ & $\begin{array}{c}\text { Cohesive } \\
\text { Energy } \\
(\mathrm{eV} / \text { atom })\end{array}$ & $\begin{array}{c}\text { Bulk } \\
\text { Modulus } \\
(\mathrm{GPa})\end{array}$ & $\begin{array}{c}\text { Vacancy } \\
\text { Energy } \\
(\mathrm{eV})\end{array}$ & $\begin{array}{c}\mathrm{p} \\
\AA^{\alpha} \\
\left(\mathrm{A}^{-1}\right)\end{array}$ & $\begin{array}{c}\lambda \\
(\AA)\end{array}$ & $\begin{array}{c}1 \\
(\AA)\end{array}$ \\
\hline $\mathrm{Ni}$ & 2.752 & 5.869 & 249.2 & 3.0 & 6 & 3.0670 & 0.763 & 0.2716 \\
\hline $\mathrm{Al}$ & 3.192 & 3.942 & 77.3 & 1.8 & 4 & 1.8756 & 1.038 & 0.3695 \\
\hline $\mathrm{Ti}$ & 3.213 & 6.270 & 121.0 & 2.0 & 6 & 2.6805 & 1.048 & 0.3728 \\
\hline $\mathrm{Cr}$ & 2.837 & 4.981 & 286.0 & 4.9 & 6 & 2.8580 & 0.6460 & 0.2300 \\
\hline $\mathrm{Cu}$ & 2.822 & 4.438 & 184.5 & 1.8 & 6 & 3.1082 & 0.7614 & 0.2710 \\
\hline
\end{tabular}

Table 8: LMTO and ECT parameters for the bcc-structured versions of the various elements used in this work.The first-principles LMTO parameters are used to determine the ECT parameters (see Ref. 21 or 23).

\begin{tabular}{|l|l|l|l|l|l|}
\hline \multicolumn{1}{|c|}{$\mathrm{jli}$} & $\mathrm{Ni}$ & $\mathrm{Al}$ & \multicolumn{1}{c|}{$\mathrm{Ti}$} & \multicolumn{1}{c|}{$\mathrm{Cr}$} & \multicolumn{1}{c|}{$\mathrm{Cu}$} \\
\hline $\mathrm{Ni}$ & 0.00000 & -0.05813 & -0.06582 & -0.02975 & 0.02085 \\
\hline $\mathrm{Al}$ & 0.08220 & 0.00000 & -0.06360 & -0.01307 & 0.05887 \\
\hline $\mathrm{Ti}$ & 0.45690 & 0.22830 & 0.00000 & 0.06579 & 0.21964 \\
\hline $\mathrm{Cr}$ & 0.20480 & -0.01637 & -0.04691 & 0.00000 & 0.02664 \\
\hline $\mathrm{Cu}$ & -0.01489 & -0.04793 & -0.05555 & -0.01016 & 0.00000 \\
\hline
\end{tabular}

Table 9: BFS parameters from LMTO results for bcc-structured alloys. The entry $\mathrm{A}_{\mathrm{ij}}$ corresponds to the BFS parameter $\Delta_{\mathrm{ij}}$ (see Ref. 7). 
We first consider the different effect that $\mathrm{Ti}$ or $\mathrm{Cr}$ additions have on the NiAl base alloy, and how these elements interact when added together, forming a quaternary alloy. Furthermore, we compare these results with a Ni-Al-Ti-Cu alloy in order to highlight the effect of different alloying additions to the surface features observed in the other systems.

We limit our discussion of results to BFS/Monte Carlo simulations, starting with the simple $\mathrm{NiAl}$ system, then the ternary Ni-Al-Ti and Ni-Al-Cr systems and finally the quaternary Ni-Al-Ti$\mathrm{Cr}$ and Ni-Al-Ti-Cu cases. A brief analysis of the numerical results of the simulations accompanies each example. The wealth of results presented here warrant a more detailed analysis based on the energies calculated with the BFS method which, as described below, distinguish between structural and chemical effects allowing for a straightforward interpretation of the observed phenomena. This will be the subject of forthcoming publications, focusing the present work on an overview of the general behavior of these multicomponent systems.

To date, the only experimental work has been limited to the binary Ni-Al system. There have been a few surface studies of pure NiAl crystals $[25,43,44]$. The results for the (110) surfaces showed no preferential segregation of Al, whereas the (100) surfaces clearly showed Al enrichment. Al segregation would be expected based on surface energy and strain energy considerations. However, Roux and Grabke [43] argue that the Ni-Al binding energy is sufficiently large to overcome these effects. More recent experimental work clearly indicates the segregation of Al to the (001) surface plane of NiAl alloys [45].

$\mathrm{NiAl}$ forms a B2 bcc-based structure, with $\mathrm{Ni}$ and $\mathrm{Al}$ atoms occupying separate interpenetrating sublattices (Fig. 2.a). Experimental and theoretical studies coincide in estimating the solubility limit of $\mathrm{Ti}$ in $\mathrm{NiAl}$ between $5 \%$ and $10 \% \mathrm{Ti}$. Beyond the solubility limit, the formation of the ternary ordered Heusler $\mathrm{Ni}_{2} \mathrm{AlTi}$ phase (Fig. 2.b) is observed [46,47]. From previous BFS calculations, the formation of Heusler precipitates has been determined by comparing the energy of formation of every possible arrangement of $\mathrm{Ni}, \mathrm{Al}$ and $\mathrm{Ti}$ atoms for a wide range of concentrations. It was found that below 5 at. $\% \mathrm{Ti}$, Ti atoms remain in solid solution in the NiAl matrix, whereas above that concentration, the formation of ordered Heusler structures is energetically favored. This behavior has been justified by means of the types of bonds formed in such atomic arrangement which clearly favors the Heusler pattern against any other ternary arrangement. For example, BFS calculations together with Monte Carlo simulations indicate the possibility of alternative ordered arrangements - a new ternary phase in the case of Ni-Al-Ti alloys [39] - but with a much 
lower probability of occurrence. Moreover, these same finite temperature Monte Carlo simulations using the BFS method for computing the energy give some indication of the influence of the temperature treatment on the final low temperature state of the alloy, where, essentially, the formation of Heusler precipitates is favored by slow cooling processes.

Experimental results performed after the simulations confirm these findings [39]. Three NiAl single crystal alloys (Ni-47Al-3Ti, Ni-45Al-5Ti and Ni-43Al-7Ti) were grown by a bridgman technique. The ingots were homogenized for $32 \mathrm{hrs}$. at $1644 \mathrm{~K}$, aged for $6 \mathrm{hrs}$. at $1255 \mathrm{~K}$ and slowly furnace cooled from the aging temperature. The purpose of this heat treatment was to produce a low temperature 'equilibrium' microstructure that would best correspond to the ground
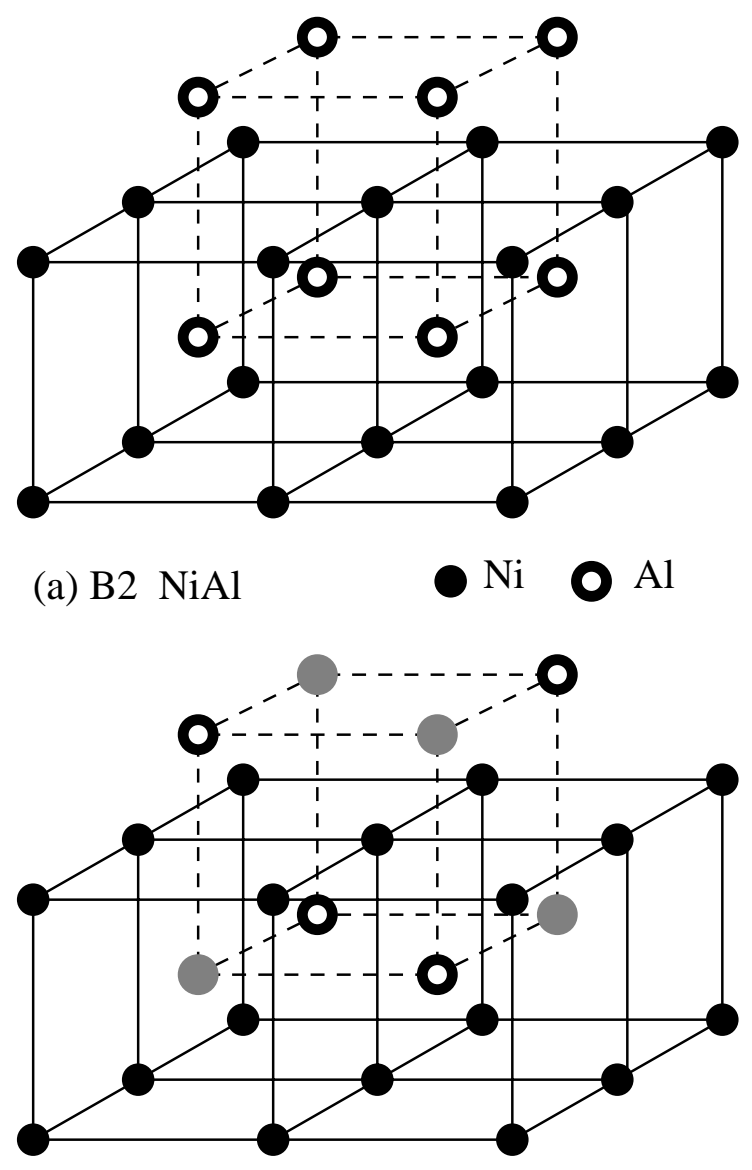

(b) Heusler phase $\mathrm{Ni}_{2} \mathrm{AlTi} \bullet \mathrm{Ni} \bigcirc \mathrm{Al} \bigcirc \mathrm{Ti}$

Fig. 2: (a) B2 structure (NiAl) and (b) Heusler phase ( $\left.\mathrm{Ni}_{2} \mathrm{AlTi}\right)$. 
state conditions modeled with the BFS method. Samples for transmission electron microscopy (TEM) were prepared from $3 \mathrm{~mm}$ diameter cylinders electro-discharge machined from the heat treated ingots. Slices sectioned from the cylinders were mechanically ground and electrochemically thinned in a twin-jet Tenupol-3 polisher. Microstructural (brightfield/darkfield) and diffraction analyses were conducted in a Phillips 400T TEM equipped with a double tilt goniometer. While the $3 \%$ alloy has a clean and featureless microstructure, the $5 \%$ alloy exhibits precipitation of high density, extremely fine, second phase particles corresponding to a $\mathrm{Ni}_{2} \mathrm{AlTi}$ phase. The $7 \%$ alloy displays well defined and coherent Heusler precipitate plates of different size.

The BFS calculation predicts the correct site preference scheme for Ti additions to NiAl, the correct lattice parameter for the $\mathrm{Ni}_{2} \mathrm{AlTi}$ alloy $(0.584 \mathrm{~nm}$, experiment; $0.583 \mathrm{~nm}, \mathrm{BFS})$ as well as the correct behavior for the lattice parameter for the whole range of $\mathrm{Ti}$ concentration in Ni-rich NiAl-Ti alloys.

The success of the BFS method in correctly describing the bulk properties and behavior of NiAl-Ti alloys establishes a reasonable degree of reliability of the method and the parameterization of $\mathrm{Ni}, \mathrm{Al}$ and $\mathrm{Ti}$ as determined by first-principles calculations.

A similar BFS analysis for NiAl-Cr once again provides results in excellent agreement with experiment. The formation of $\alpha-\mathrm{Cr}$ precipitates is observed both experimentally and theoretically beyond 1-2 at. \% Cr [48]. Moreover, the BFS calculations, as well as the finite temperature Monte Carlo simulations using BFS for computing the energy, also agree with experiment with respect to the orientation of the $\alpha-\mathrm{Cr}$ precipitates in the $\mathrm{NiAl}$ matrix as well as in the formation of additional phases.

Experimental results for NiAl-Cr alloys [48] indicate the formation of a two phase alloy, confirming the theoretical predictions. The agreement between the BFS results and experiment raise the necessary confidence in the validity of the method and the $\mathrm{Cr}$ parameterization used.

While no similar studies exist for $\mathrm{Cu}$ in Ni-rich $\mathrm{NiAl}$ alloys, we have included such a case in this work with the goal of examining the interaction between several types of alloying additions and their influence both in the bulk and surface properties of these multicomponent alloys.

Given the complexity of the systems at hand, it is necessary, in this first attempt to describe these systems, to simplify the analysis by concentrating on just one aspect of the problem, namely the difference between bulk and surface composition, leaving for future studies the details in the process of surface formation and segregation to the surface. 
To achieve this goal, we approach the simulations in the following fashion: 1) a free-standing thin film with a predetermined crystallographic structure is defined (in our case, a bcc lattice terminating in (110) surfaces on both sides of the film), 2) a composition is determined thus fixing the number of atoms of each atomic species, such that every site in the film will be occupied (i.e. no vacancies are allowed in this calculation), 3) an initial, random distribution is determined, corresponding to an elevated temperature state, 4) a Monte Carlo/Metropolis calculation (see Section 4.b) at different temperatures is carried out until the energy of the system converges to a constant value. In all the calculations shown in this work, the temperature is steadily lowered in $100 \mathrm{~K}$ steps, giving the atoms the chance to satisfy ordering conditions typically found in these alloys, or some new ones solely due to the presence of a surface.

The simulations shown correspond to a process where a sequence of decreasing temperatures (the 'cascade') is chosen [39], where the system is allowed to equilibrate sequentially at each temperature (simulating the 'slow cooling' of the actual alloy, starting from high temperature disordered solution, but always within the framework of a bcc-film). Because the lattice parameter and all other properties are assumed to be independent of temperature, the temperature enters into the calculation only through the Metropolis criterion. After the system has achieved equilibrium (based on the total energy), the simulation is allowed to proceed further while various properties of the system are computed and averaged. These include the average energy of the cell, the specific heat and bond correlations.

While these simulations do not attempt to mimic the detailed dynamics of the equilibration process, they do offer a qualitative view of the effects of rapid versus slow cooling of the system. The temperature treatment (that is, the size of the steps between the various temperatures considered in the cascade) is of critical importance in determining the final state of the system. Slow cooling results in a highly ordered low temperature state, while rapid cooling results in a system with grain structure.

As mentioned before, the geometry to be used is the B2 structure with periodic boundary conditions in directions perpendicular to the (110) faces of the film (Fig. 3). The film thickness which corresponds to 14 atomic planes- ranges from 2.1 to $2.2 \mathrm{~nm}$, depending on the number and type of atomic species included $(2.136 \mathrm{~nm}$ for Ni-33Al-34Cr, $2.137 \mathrm{~nm}$ for Ni-48Al-2Ti, 2.146 $\mathrm{nm}$ for Ni-45Al-5Ti, $2.156 \mathrm{~nm}$ for Ni-40Al-10Ti, $2.147 \mathrm{~nm}$ for Ni-23Al-10Ti-34Cr and 2.183 nm for Ni-24Al-24Ti-2Cu). 


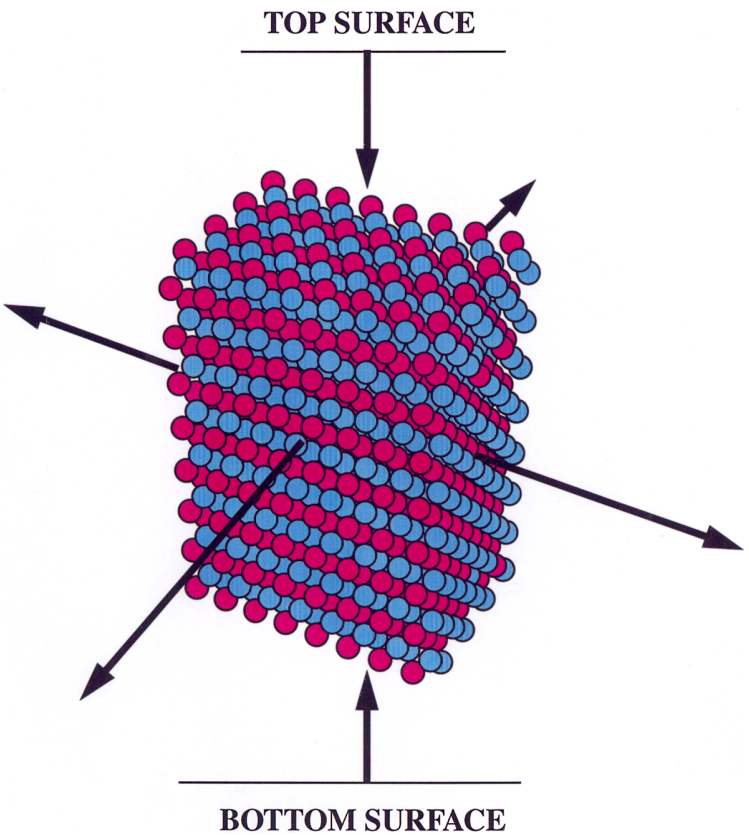

Figure 3.-Thin film computational binary cell: the cell contains 1024 atoms and is periodic in the two directions perpendicular to the (110) surfaces (top and bottom planes). 


\section{5.a. NiAl}

Fig. 4 shows the results for a binary NiAl alloy, comparing a bulk cell (Fig. 4.a)(periodic in all three directions) displaying perfect B2 ordering, with a similar size cell corresponding to a portion of a film (Fig. 4.b) (periodic in only two directions, perpendicular to the top and bottom planes). For clarity, we also show a stretched version of each cell in order to clearly distinguish the differences in atomic distributions between the bulk and film cells. The film cell is stretched along the axis perpendicular to the surface. Also, in order to give some indication on the variety of possible outcomes of a Monte Carlo calculation in terms of the initial state as well as the temperature treatment, the results of two separate simulations are shown for the case of the film.

The most salient feature in the film structure is the formation of an Al surface plane on both sides of the film, at the expense, mostly, of $\mathrm{Al}$ atoms that were originally in planes close to the surface. Very little change in composition is observed in planes near the center of the film which, of course, simulate a bulk environment. The formation of an Al plane at the surface is in agreement with recent experimental results [45] and it can be easily explained in terms of the larger size of the $\mathrm{Al}$ atoms as well as by the low surface energy of pure $\mathrm{Al}$ surfaces.

A way to roughly measure the changes in composition between the bulk and the film cell is

given by the coordination matrix, whose element $c_{i j}$ indicates the probability that an atom of species $i$ has an atom of species $j$ as a nearest neighbor. Defined that way, a perfect bulk B2 structure would be described by

$$
\left[\begin{array}{ll}
0 & 1 \\
1 & 0
\end{array}\right]
$$

with the first column and row assigned to $\mathrm{Ni}$ and the second to Al. Similarly, the ideal Heusler coordination matrix (i.e. $\mathrm{Ni}_{2} \mathrm{AlTi}$ ) is

$$
\left[\begin{array}{lll}
0.0 & 0.5 & 0.5 \\
1.0 & 0.0 & 0.0 \\
1.0 & 0.0 & 0.0
\end{array}\right],
$$

indicating that $\mathrm{Al}$ and $\mathrm{Ti}$ are located in one sublattice (so that they only have $\mathrm{Ni}$ atoms as nearest neighbors) and $\mathrm{Ni}$ atoms in the other sublattice (having equal number of $\mathrm{Al}$ and $\mathrm{Ti}$ atoms as near- 

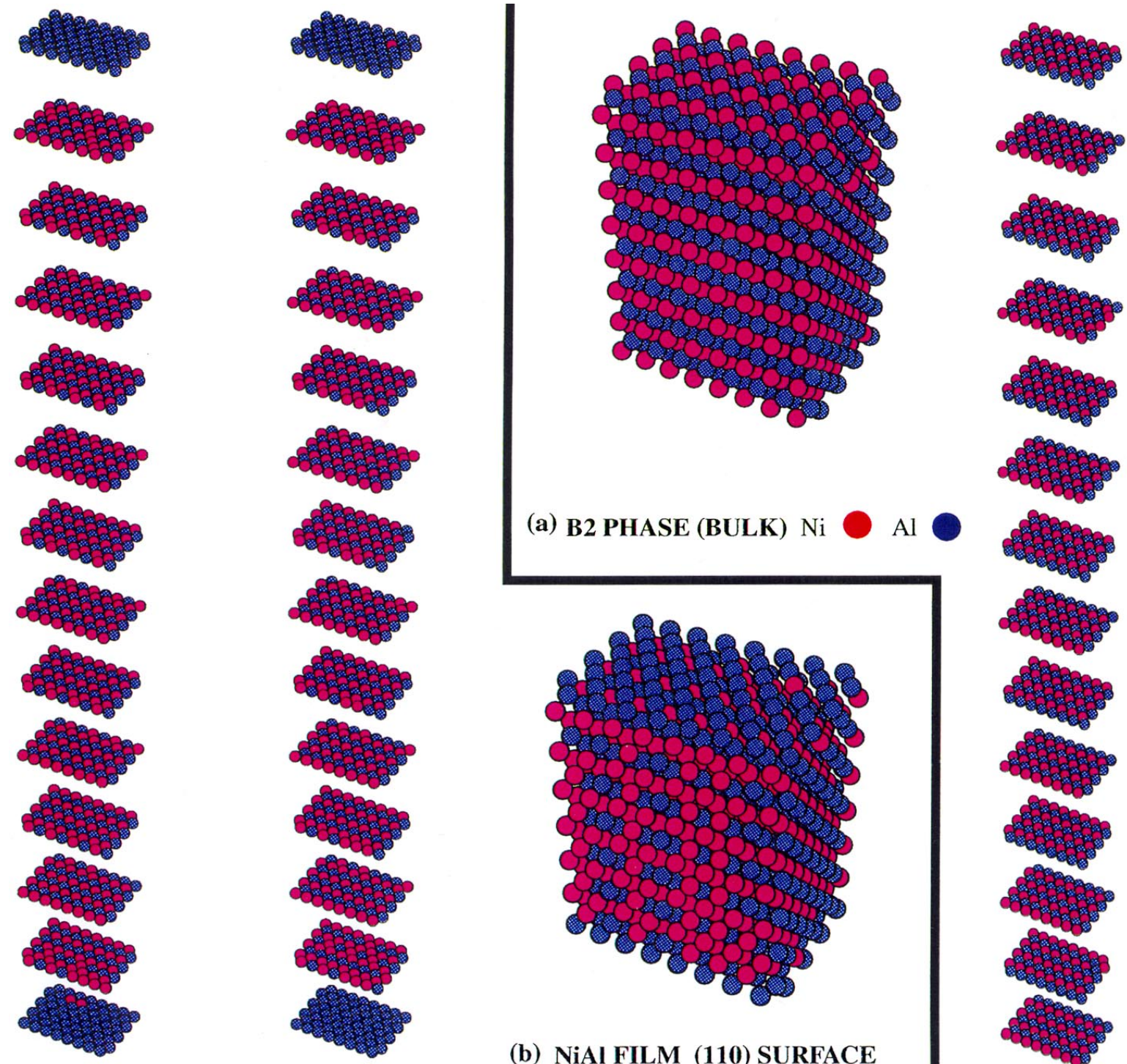

(a) B2 PHASE (BULK) $\mathrm{Ni} \bigcirc \mathrm{Al}$
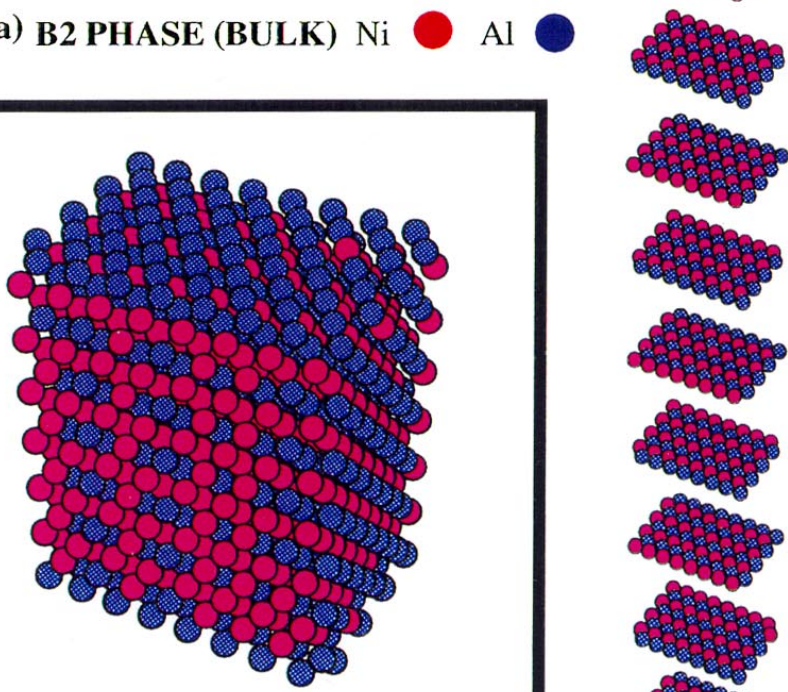

(b) NiAI FILM (110) SURFACE

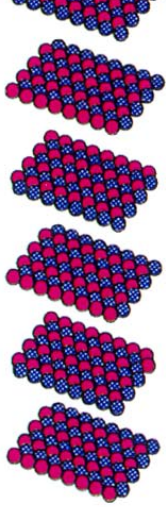

Figure 4.- (a) Thin film computational cell (1024 atoms) for NiAl: the first two columns represent the stretch cells resulting from two different Monte Carlo simulations (different initial state, same temperature treatment). The compact cell corresponds to the second stretched cell. (b) B ulk cell for NiAl with the same number atoms. 
est neighbors). For the film cell, the coordination matrix at the final stage of the Monte Carlo simulation is

$$
\left[\begin{array}{ll}
0.27 & 0.73 \\
0.37 & 0.63
\end{array}\right]
$$

indicating the formation of mostly pure $\mathrm{Al}$ surface planes by the large probability (0.63) of Al-Al nearest-neighbor pairs (mostly in the top two planes on each side of the film) and some Ni-Ni nearest-neighbor pairs due to the $\mathrm{Ni}$ antistructure atoms abundant in Al-poor regions of the film.

\section{5.b. $\mathrm{NiAl+Ti}$}

The addition of $\mathrm{Ti}$ introduces some changes in the composition of the planes close to the surfaces. These examples are shown in Fig. 5 where stretched bulk and film cells are shown for three NiAl-Ti alloys: Ni-47.7Al-2.3Ti, Ni-45Al-5Ti and Ni-40.2Al-9.8Ti. The final state coordination matrices are (the successive rows and columns correspond to $\mathrm{Ni}, \mathrm{Al}$ and $\mathrm{Ti}$, respectively)

$$
\left[\begin{array}{lll}
0.2045 & 0.6705 & 0.1250 \\
0.3114 & 0.6382 & 0.0504 \\
0.7500 & 0.2500 & 0.0000
\end{array}\right]
$$

for $\mathrm{Ni}-47.7 \mathrm{Al}-2.3 \mathrm{Ti}$,

$$
\left[\begin{array}{lll}
0.2614 & 0.4659 & 0.2727 \\
0.2344 & 0.6224 & 0.8432 \\
0.7143 & 0.2857 & 0.0000
\end{array}\right]
$$

for $\mathrm{Ni}-45 \mathrm{Al}-5 \mathrm{Ti}$ and

$$
\left[\begin{array}{lll}
0.2143 & 0.4643 & 0.3214 \\
0.2240 & 0.6224 & 0.1535 \\
0.7167 & 0.2833 & 0.0000
\end{array}\right]
$$

for Ni-40.2Al-9.8Ti. The first example, Ni-47.7Al-2.3Ti, reproduces the main feature of the previous case $(\mathrm{NiAl})$, consisting of the segregation of $\mathrm{Al}$ atoms to the surface, a feature that is common to all cases. While the bulk cell contains the Ti atoms in solid solution in the NiAl matrix, the thin 


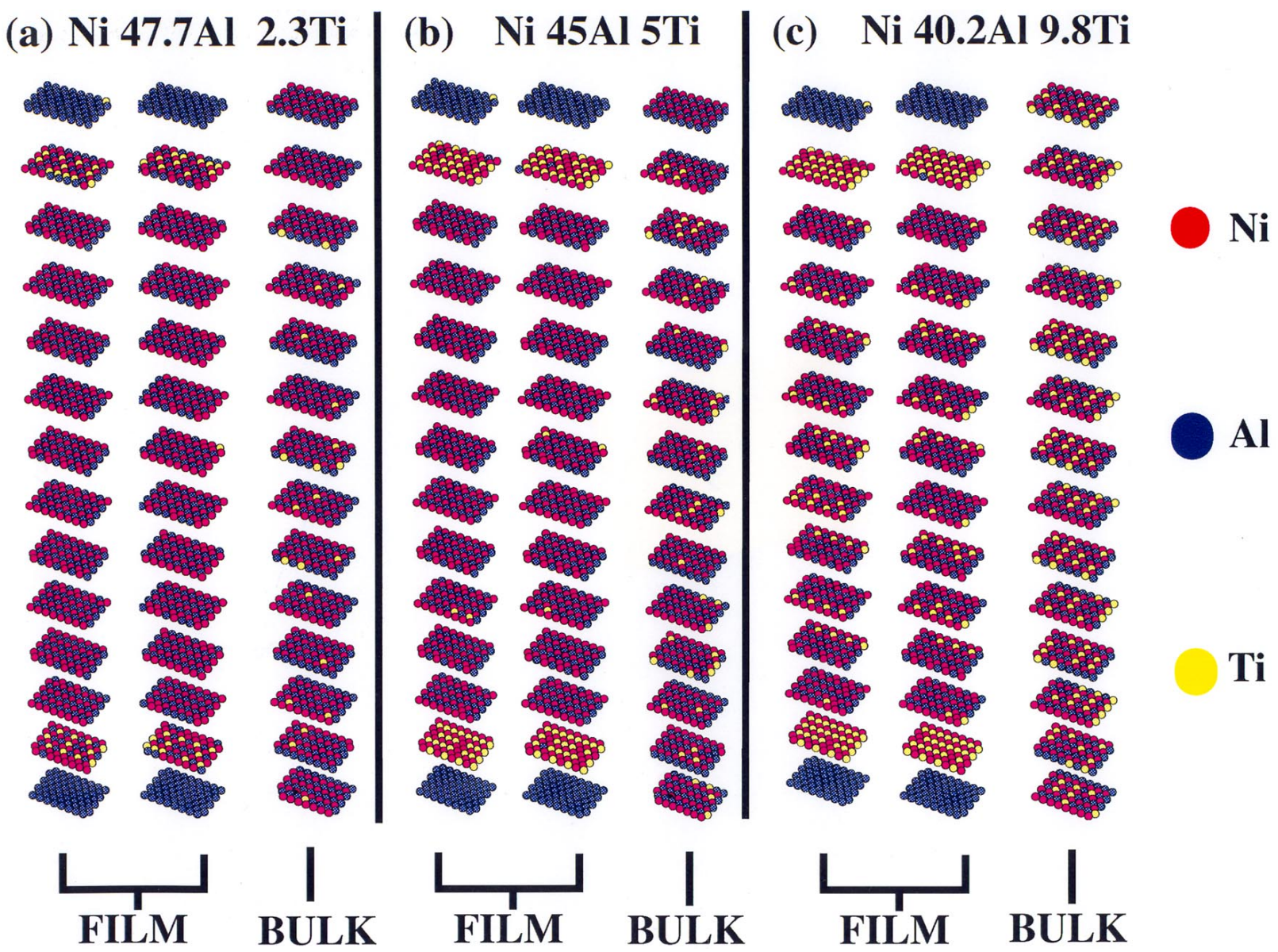

Figure 5.- Results of Monte Carlo simulations for three NiAl-Ti alloys: (a) Ni-47.7Al-2.3Ti, (b) Ni-45Al-5Ti and (c) Ni-40.2Al-9.8Ti. In each case, the first two stretched cells indicate the results of two different simulations while the third cell shows the corresponding bulk cell (periodic in all three directions). 
film displays additional segregation of all $\mathrm{Ti}$ atoms to the plane below the surface, with hints of a preferential ordering pattern (Fig. 5.a).

This effect is more noticeable in the Ni-45Al-5Ti alloy: segregation of $\mathrm{Ti}$ atoms to the plane below the surface is again observed, with the additional feature that an ordered Ni-Al-Ti 2- (or perhaps 3-) layer alloy is thus defined. A few Ti atoms remain in solid solution in the bulk of the film (Fig. 5.b). Above the solubility limit, the third example (Fig. 5.c) repeats the previously described features with the addition of the formation of Heusler precipitates - as expected - in the bulk. The results of two different simulations also suggest that the second plane below the surface remains a pure $\mathrm{Ni}-\mathrm{Al}$ plane, with the formation of Heusler precipitates restricted to the center of the film. This explains the decrease in the $c_{11}=c_{N i N i}$ element in the coordination matrix for this alloy in contrast to that for Ni-45Al-5Ti: the larger concentration does not reflect into additional coordination, with most of the $\mathrm{Ni}-\mathrm{Ni}$ bonds being those between atoms in the second and third plane below the surface plane.

The formation of Heusler precipitates is to be expected given the bulk environment at the center of the film. So is the segregation of Al to the surface plane as discussed earlier. The most salient new feature in this simulation is therefore the formation of a surface $\mathrm{Ni}_{2} \mathrm{Al}_{3} \mathrm{Ti}$ alloy whose existence, of course, should be verified by relaxing some of the limitations imposed in this calculation where no relaxation of atomic planes or individual atoms, nor surface reconstruction, were allowed.

To complete the discussion on Ni-Al-Ti alloys, we show the $\mathrm{Ni}_{2} \mathrm{AlTi}$ Heusler alloy (Fig. 6). The bulk cell displays the perfect Heusler ordering while the film cell once again shows most of the features already seen in the Ni-40.2Al-9.8Ti case: segregation of Al to the surface, of Ti to all $\mathrm{Al}$ sites in the second plane and a large number of Heusler precipitates toward the center of the film. In addition, there is a noticeable presence of $\mathrm{Ti}$ in the top plane and, once again, a Ti depletion in the second plane below the surface, reaffirming the previously discussed formation of a ternary surface alloy in the top three planes of the film, characterized by Ni-depletion of the top plane, resulting in a Ti-Al surface, Ti-enrichment (substituting for Al) in the second plane and Tidepletion for the third plane, just before the film recovers essentially the bulk structure. 


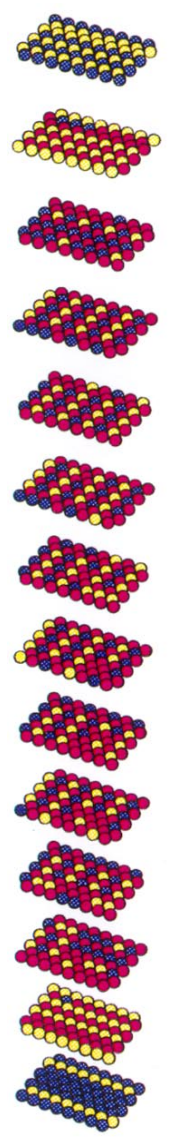

Ni-25Al-25Ti

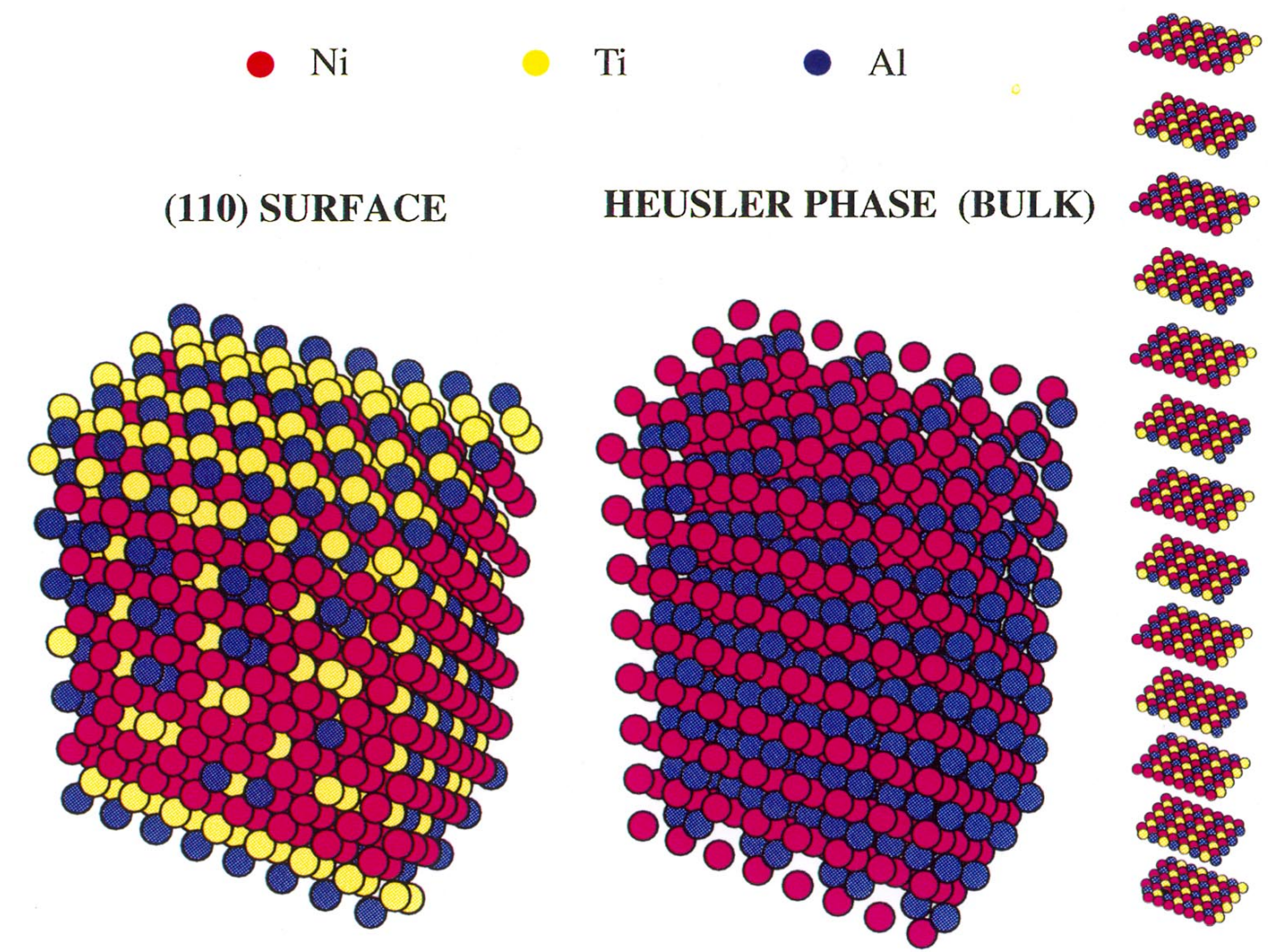

Figure 6. - (a) Thin film cell and (b) bulk cell for Ni-25Al-25Ti. 


\section{5.c. $\mathrm{NiAl}+\mathrm{Cr}$}

$\mathrm{Cr}$ additions to NiAl display a different behavior than that seen in NiAl-Ti alloys. The solubility limit of $\mathrm{Cr}$ in $\mathrm{NiAl}$ is between 1 and 2 at. \% Cr. The example analyzed in this work, Ni-33Al$34 \mathrm{Cr}$, was chosen due to the availability of experimental data for the bulk alloy. TEM analysis shows the clear formation of $\alpha$-Cr precipitates, a feature that is also clearly predicted by the BFS calculations shown in Fig. 7.a, where a slow cooling of the sample induces the formation of an $\alpha$ Cr precipitate, with the same orientation found experimentally.

The experimental results also show the formation of dislocation lines due to the misfit between the $\alpha-\mathrm{Cr}$ precipitate and the $\mathrm{NiAl}$ matrix. The formation of $\mathrm{Cr}$ precipitates is therefore to be expected in Ni-rich alloys with $\mathrm{Cr}$ additions above the solubility limit. BFS calculations confirm this expectation [49] as well as the presence of a third, $\gamma-\mathrm{Ni}$ phase for a certain range of $\mathrm{Al}$ and $\mathrm{Cr}$, in agreement with the phase diagram for these systems. The corresponding film (Fig. 7.b) shows three distinctive features: segregation of Al to the surface plane (similar to the behavior observed in NiAl-Ti alloys), the formation of a $\mathrm{Cr}$ precipitate immediately below the surface and some segregation of $\mathrm{Cr}$ to the otherwise pure $\mathrm{Ni}$ plane in the face of the film opposite to that where the $\alpha-\mathrm{Cr}$ precipitate forms. The site preference of $\mathrm{Cr}$ for $\mathrm{Al}$ sites in $\mathrm{NiAl}$ alloys favors short range order in what otherwise could be considered a ternary ordered Ni-Al-Cr surface alloy, in a similar fashion to that found in $\mathrm{Ni}-\mathrm{Al}-\mathrm{Ti}$ in our previous discussion.

The fact that the $\mathrm{Cr}$ precipitate forms only on one side of the film instead of partitioning between the two sides could be a result of the limited size of the computational cell and the uneven presence of $\mathrm{Cr}$ atoms in the initial random distribution, which could favor nucleation of the Cr precipitate on a specific side of the film.

\section{5.d. $\mathrm{NiAl}+\mathrm{Cu}$}

Finally, we discuss $\mathrm{Cu}$ additions to NiAl. The BFS calculations indicate a weak $\mathrm{Cu}$ preference for $\mathrm{Al}$ sites, with just $0.02 \mathrm{eV}$ energy difference between the $\mathrm{Cu}(\mathrm{Al})$ and the $\mathrm{Cu}(\mathrm{Ni}) \mathrm{Al}$ cases where $\mathrm{A}(\mathrm{B})$ indicates an atom $\mathrm{A}$ in a $\mathrm{B}$ site and $\mathrm{A}(\mathrm{B}) \mathrm{C}$ indicates the same situation but with the displaced $\mathrm{B}$ atom occupying a $\mathrm{C}$ site. However, the site preference for $\mathrm{Al}$ sites is more pronounced with increasing concentration, thus predicting a dominant $\mathrm{Cu}(\mathrm{Al})$ substitutional scheme with few $\mathrm{Ni}$ antistructure atoms. The weak site preference of $\mathrm{Cu}$ additions can be explained by the competition between the new, energetically favorable, $\mathrm{Cu}-\mathrm{Al}$ bonds created when $\mathrm{Cu}$ substitutes for $\mathrm{Ni}$ 
(a) Ni-33Al-34Cr BULK

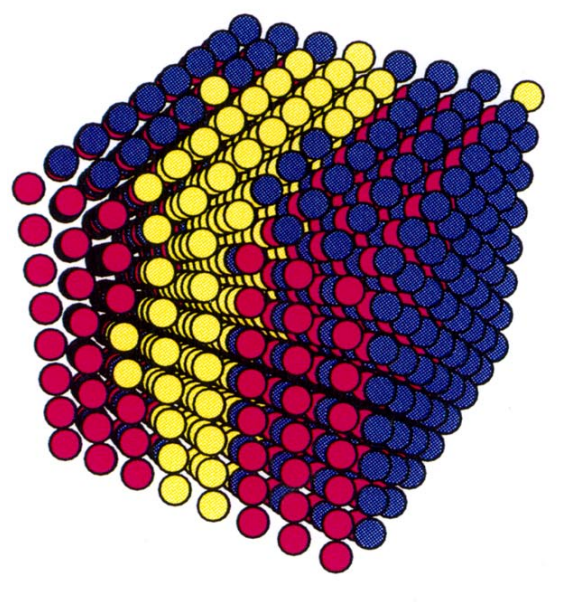

- $\mathrm{Ni} \mathrm{Al} \quad \mathrm{Cr}$
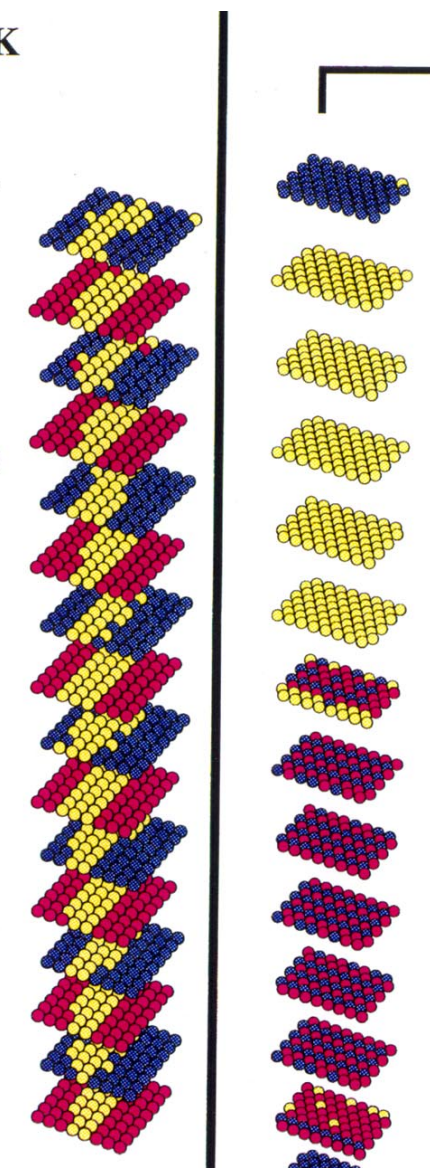

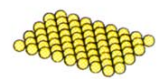

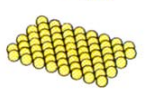

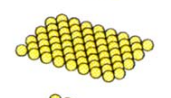

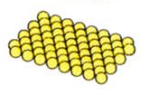

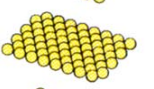
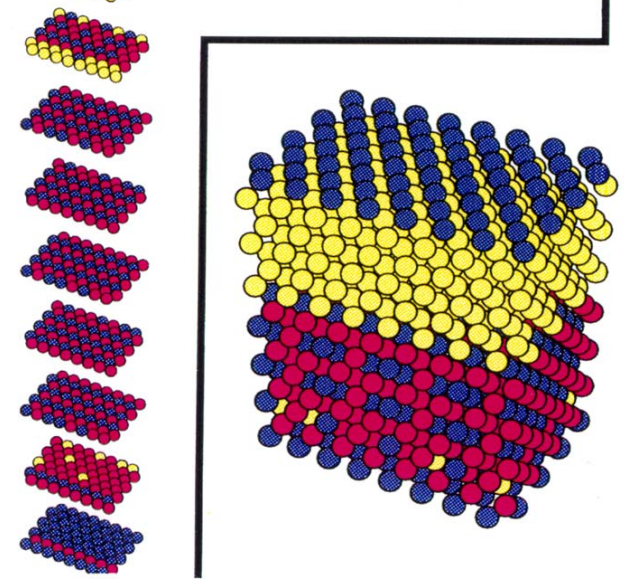

(b) Ni-33Al-34Cr FILM
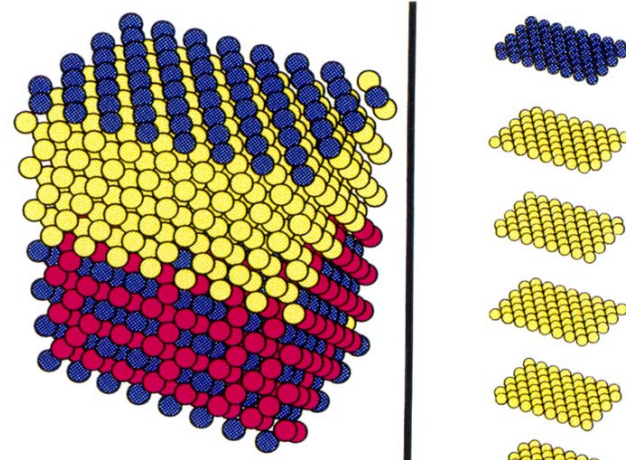

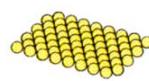

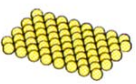

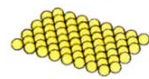

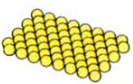

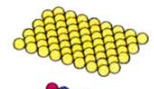
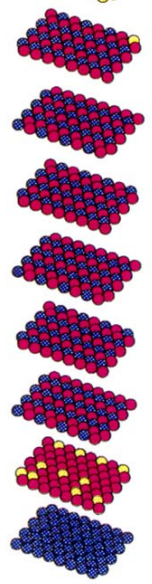

Figure 7.- (a) B ulk cell for Ni-33Al-34Cr and (b) thin film cell for the same composition (the results of two different simulations are shown). 
and the corresponding number of $\mathrm{Ni}-\mathrm{Al}$ bonds eliminated in the process, which makes the $\mathrm{Cu}(\mathrm{Ni}) \mathrm{Al}$ substitutional defect quite close in energy to the preferable $\mathrm{Cu}(\mathrm{Al})$ case.

Fig. 8 shows some characteristic results for $\mathrm{Cu}$ in NiAl. Simulations were performed for several films (a computational cell of 960 atoms) containing different $\mathrm{Cu}$ concentrations. The effect seen is more pronounced in films with the (100) orientation, therefore we show results for that face only.

The simulations predict two types of behavior: the state with lowest energy consists of an $\mathrm{Al}$ surface plane with all the available $\mathrm{Cu}$ atoms occupying sites in this plane. A Ni/Al/Ni stacking pattern follows, where all the Al planes contain some antistructure Ni atoms. This configuration the one with the lowest energy for all $\mathrm{Cu}$ concentrations - competes with one that is very close in energy but that displays a quite different concentration pattern: the surface plane is pure Al, followed by a one-plane mix of all three elements, in turn followed by the bulk Ni/Al/Ni pattern. The existence of the two consecutive Ni-rich planes is afforded due to the presence of all three elements in one of them. The simulations reach both final states, as is to be expected due to their small energy difference. The likelihood of reaching the high energy state (characterized by a pure $\mathrm{Al}$ surface plane) decreases with increasing $\mathrm{Cu}$ concentration: it is observed that above 3 at. \% $\mathrm{Cu}$, all simulations yield the $\mathrm{Al}+\mathrm{Cu}$ surface plane configuration as the final state.

One simple explanation of this dual behavior can be given in terms of strain effects. Both $\mathrm{Cu}$ and $\mathrm{Al}$ show segregation tendencies in $\mathrm{Ni}$ alloy, however the larger size of $\mathrm{Al}$ and its predominance in low $\mathrm{Cu}$ concentration systems favors then the formation of a pure $\mathrm{Al}$ plane with the $\mathrm{Cu}$ atoms having no chance of segregating to this plane, and are thus relegated to the first plane below. As the $\mathrm{Cu}$ concentration increases (and the number of $\mathrm{Al}$ atoms decreases), the competition between both species reaches the point where $\mathrm{Cu}$ atoms segregate to the surface plane thus yielding the observed ordering pattern, which forces the presence of antistructure $\mathrm{Ni}$ atoms in $\mathrm{Al}$ planes. The small energy difference between the two structures, just $0.03 \mathrm{eV} /$ atom indicates that the most likely surface composition scheme could actually be a mixture of both cases. Still, the lower energy state consists basically of the one expected for binary Ni-rich alloys (Al-rich surface plane followed by alternating $\mathrm{Ni}$ and $\mathrm{Al}$ planes with the excess $\mathrm{Ni}$ atoms appearing as antistructure atoms in the $\mathrm{Al}$ planes), with the addition of $\mathrm{Cu}$ atoms in the surface. 


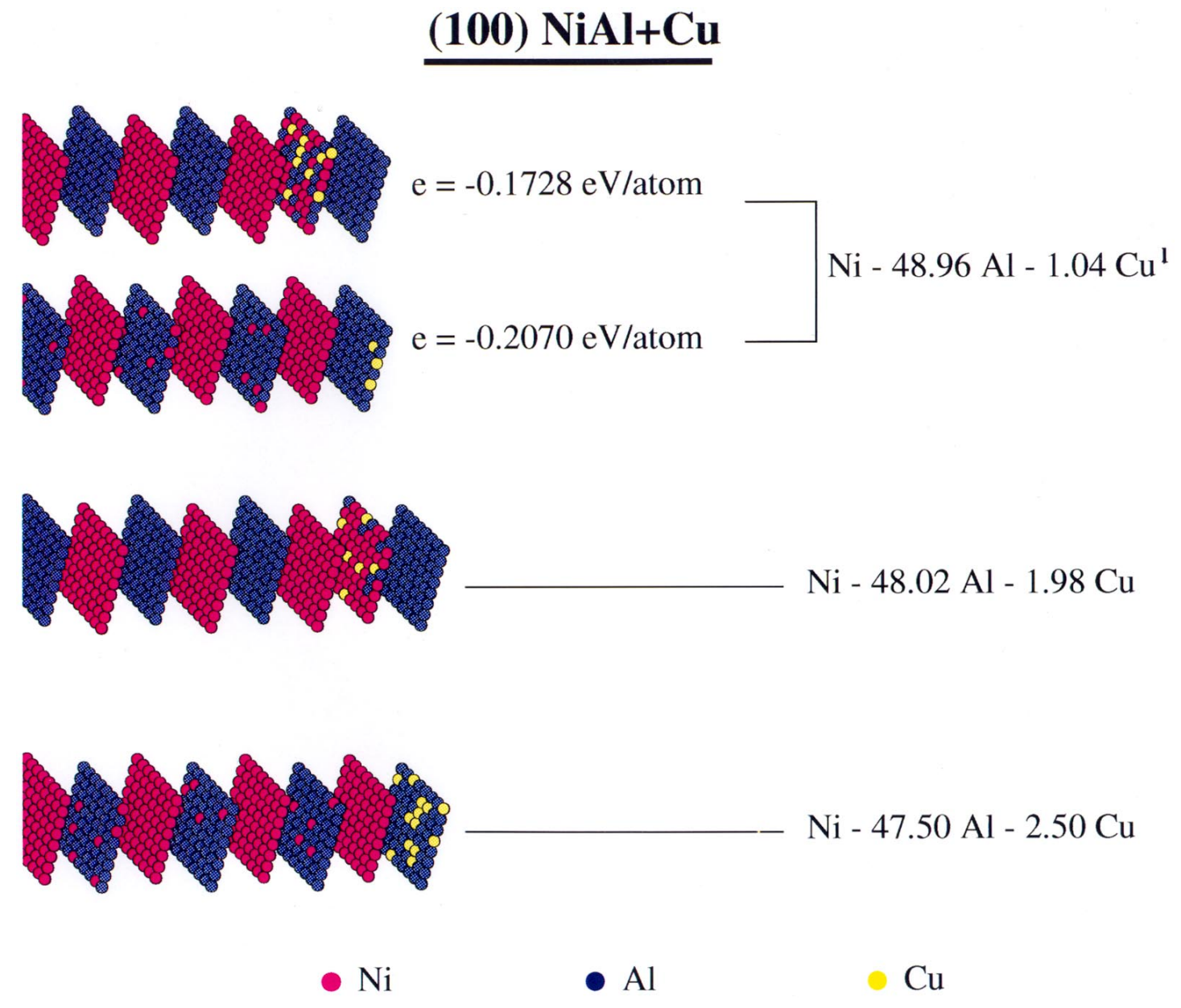

Figure 8.-Thin film cell for (100) Ni-Al-Cu films. The top two films correspond to the two lowest energy configurations of Ni-48.96Al-1.04 $\mathrm{Cu}$. The second case (third row) is Ni-48.02Al-1.98, showing how a metastable state can be reached as a result of the Monte Carlo simulation. Finally the third case (fourth row), which corresponds to a Ni-47.50Al-2.50 $\mathrm{Cu}$ (110) film indicates typical results for larger $\mathrm{Cu}$ concentrations. 


\section{5.e. $\mathrm{NiAl}+(\mathrm{Ti}, \mathrm{Cr}) /(\mathrm{Ti}, \mathrm{Cu})$}

We now consider the quaternary systems NiAlTiX $(X=\mathrm{Cu}, \mathrm{Cr})$ contrasting the predicted behavior with that already described for the ternary systems. Fig. 9.a shows results for Ni-23Al10Ti-34Cr, whose composition was chosen due to the already known separate behavior of Ti and $\mathrm{Cr}$, as discussed in previous paragraphs.

The formation of a nearly pure Ti surface on top of the expected $\mathrm{Cr}$ precipitate continues the trend already observed in the Ni-40Al-10Ti case, where Ti segregated to the plane below the surface. The lower $\mathrm{Al}$ concentration in the current system also facilitates the formation of this $\mathrm{Ti}$ plane. Otherwise, all known features are also found on the other side of the film: formation of an Al surface plane, a Ti-Ni mix immediately below, and the remaining $\mathrm{Ti}$ atoms displaying some short range ordering in the $\mathrm{Ni}-\mathrm{Al}$ bulk planes of the film. This Ti-Al surface plane forms at the expense of a more predominant presence of $\mathrm{Ti}$ in the NiAl matrix - seen in the ternary case- so that the Heusler precipitates are virtually nonexistent in this quaternary alloy.

It is interesting to note that a pure $\mathrm{Ni}$ phase separates the $\mathrm{Cr}$ precipitate from the $\mathrm{Ni}-\mathrm{Al}$ matrix, which is consistent with the three-phase bulk alloy found at that concentration. This example is useful in the sense that it clearly distinguishes between 'bulk alloy' features (i.e. formation of the Cr precipitate, $\mathrm{Ti}$ in solid solution in the alloy, formation of new Ni phases) and 'surface alloy' features (i.e. formation of the Ni-Al-Ti phase on one face of the film and formation of the Ti surface plane on the side with the $\mathrm{Cr}$ precipitate). Obviously, a much larger number of examples should be studied before making any definite statement regarding the observed behavior, carefully considering the dependence of the final results on several intervening factors. Moreover, some of the restrictions imposed in this calculation should be relaxed if meaningful comparisons with future experiments are ever made.

Nonetheless, the goal of the present study, which is to test the ability of the BFS method to provide information on complex systems intractable by other techniques, has been realized, regardless of the limitations imposed, as none of them are based on shortcomings of the method itself. All the restrictions imposed in this calculation were made for the sake of simplicity and to clarify the discussion of results: with such complex systems, it becomes strictly necessary to freeze certain degrees of freedom in order to gain understanding on simple, basic effects that ultimately determine the behavior of the system. 


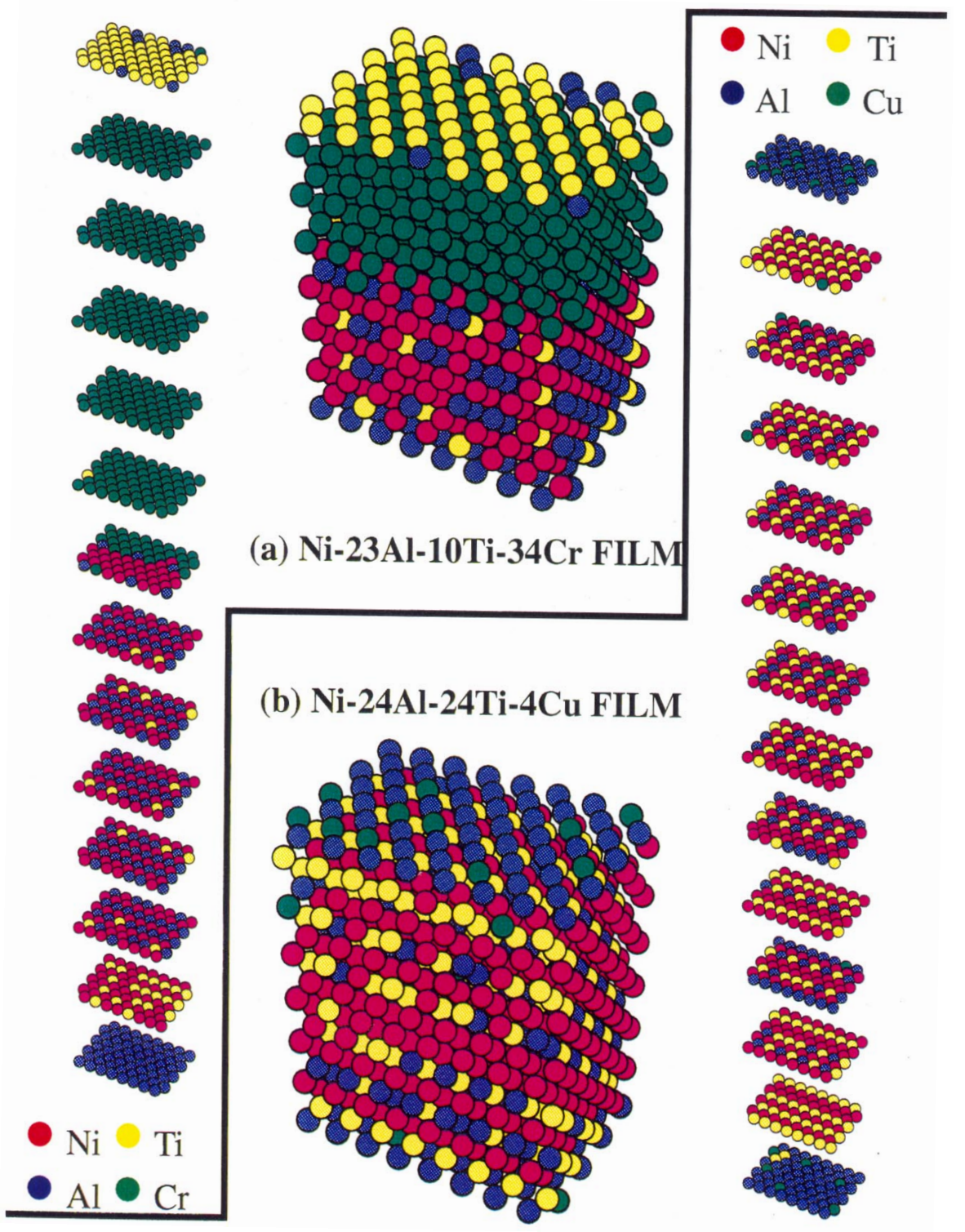

Figure 9.- (a) Monte Carlo results for thin film cells of Ni-23Al-10Ti-34C $r$ and (b) Ni-24Al-24Ti-4Cu. 
By fixing the type of crystallographic structure - perhaps one of the most serious limitations we are severely limiting the study to what might probably be an unrealistic situation. In the particular examples studied, there is enough experimental evidence to support and justify the use of such restriction, but this might not be true in general. However, imposing such constraint enables us to single out other features, like the influence of more than one alloying addition in the same basic system.

This is clearly shown in the last example to be discussed in this paper: Ni-24Al-24Ti-2Cu shown in Fig. 9.b. This is basically a Heusler alloy with small $\mathrm{Cu}$ additions. The bulk alloy, consistent with the site preference scheme of $\mathrm{Cu}$ in Ni-Al-Ti alloys [50] indicates that $\mathrm{Cu}$ will occupy Al sites forming a quaternary ordered structure. The film, however, clearly exhibits a quite different behavior, where the segregation of $\mathrm{Cu}$ to the Al-surface planes is clearly dominant. Otherwise, the structure of the film is basically identical to that previously seen in Fig. 7, indicating that the surface effect, i.e. segregation of $\mathrm{Cu}$ to the top plane, is 'decoupled' from any effect that $\mathrm{Cu}$ might have in the bulk structure of this system.

\section{Conclusions}

Surface and interfacial segregation are phenomena with great technological importance [51]. They affect both the chemistry of surfaces and the strength of interfaces. Some important affected areas include crystal growth, catalysis, semiconducting interfaces and the mechanical strength of solids affected by grain boundary segregation. Although theory has lagged behind experiment, now first-principles and semi-empirical methods have arrived at a point where they can aid in understanding and supplementing experiment, and be used for prediction of properties. In this paper, we present predictions using a relatively new, semiempirical method, BFS, of the surface and bulk properties of multi-component, alloy thin films for which BFS is particularly simple and applicable.

The choice of alloys studied in this work was made so that a close link with the existing body of experimental data was kept and a natural progression from binary to quaternary alloys was followed. The possibilities, even for such a small number of elements, are endless. The examples studied are limited in number and scope to extract from these results a general idea of the dominant behavior. However, the main goal of this work is to show that the methodology exists to 
attempt such a study and that the insight that could be gained from the theoretical and numerical analysis shown should be proof that a comprehensive approach, including atomistic modeling and experimental work, should be attempted in order to reasonably deal with such complex systems as the ones discussed here. It is our hope that these results will also serve to inspire further first-principles, experimental and theoretical studies. We have also presented evidence of the accuracy of BFS in predicting segregation and segregation related phenomena. Work is currently in progress for providing further thermodynamic approaches for studying multi-component systems with BFS.

\section{References}

[1] L. Z. Mezey and W. Hofer, Surf. Sci. 352-354 (1996) 15; W. Hofer and L. Z. Mezey, Fresenius J. Anal. Chem. 353 (1995) 631; I. Dasgupta and A. Mookerjee, J. Phys. : Condens. Matter 8 (1996) 4125.

[2] L. Zhang, M. Kuhn and U. Diebold, Surf. Sci. 371 (1997) 223.

[3] S. Mizuno, H. Tochihara, A. Barbieri and M. A. Van Hove, Phys. Rev. B 51 (1995) 1969.

[4] H. L. Davis and J. R. Noonan, Phys. Rev. Lett. 54 (1985) 566; S. P. Chen, A. F. Voter and D. J. Srolovitz, Phys. Rev. Lett. 57 (1986) 1308; W. D. Roos, J. du Plessis and G. N. van Wyk, J. Vac. Sci. Technol. A 14 (1996) 1648; H. Graupner, L. Hammer, K. Mueller and D. M. Zehner, Surf. Sci. 322 91995) 103; M. Polak, J. Deng and L. Rubinovich, Phys. Rev. Lett. 78 (1997) 1058; R. P. Blum, D. Ahlbehrendt and H. Niehus, Surf. Sci. 366 (1996) 107.

[5] N. -H. Heo, Acta mater. 44 (1996) 1581.

[6] T. Flores, S. Junghans and M. Wuttig, Surf. Sci. 371 (1997) 1, ibid. Surf. Sci. 371 (1997) 14. 
[7] G. Bozzolo, J. Ferrante and R. Kobistek, J. Computer-Aided Mater. Design 1 (1995)305.

[8] S. M. Foiles, M. I. Baskes and M. S. Daw, Phys. Rev. B 33 (1986) 7983.

[9] M. W. Finnis and J. E. Sinclair, Philos. Mag. A 50 (1984) 45.

[10] G. Bozzolo and J. Ferrante, Phys. Rev. B 45 (1992) 12191.

[11] G. Bozzolo and J. Ferrante, Scripta Metall. 26 (1992) 1275.

[12] G. Bozzolo and J. Ferrante, Ultramicroscopy 42/44 (1992) 55.

[13] B. Good, G. Bozzolo and J. Ferrante, Phys. Rev. B 48 (1993) 18284.

[14] R. Kobistek, G. Bozzolo, J. Ferrante and H. Schlosser, Surf. Sci. 307/309 (1994) 390.

[15] L. Pleth Nielsen, F. Besenbacher, I. Stensgaard, E. Laegsgaard, C. Engdhal, P. Stoltze, K. W. Jacobsen and J. K. Norskov, Phys. Rev. Lett. 71 (1993) 754.

[16] G. Bozzolo, R. Ibanez-Meier and J. Ferrante, Phys. Rev. B 51 (1995) 7207; G. Bozzolo, J. Ferrante and R. Ibanez-Meier, Surf. Sci. 352-354 (1996) 577.

[17] G. Bozzolo and J. Ferrante , J. Computer-Aided Mater. Design 2 (1995) 113

[18] G. Bozzolo, B. Good and J. Ferrante, Surf. Sci. 289 (1993) 169.

[19] G. Bozzolo and J. Ferrante, Phys. Rev. B 50 (1994) 5971.

[20] L. Vegard, Z. Phys. 5 (1921) 17.

[21] J. R. Smith, T. Perry, A. Banerjea, J. Ferrante and G. Bozzolo, Phys. Rev. B 44 (1991) 6444;

G. Bozzolo, J. Ferrante and A. Rodriguez, J. Computer-Aided Mater. Design 1 (1993) 285.

[22] J. H. Rose, J. R. Smith and J. Ferrante, Phys. Rev. B 28 (1983) 1835.

[23] A. M. Rodriguez, G. Bozzolo and J. Ferrante, Surf. Sci. 289 (1993) 100.

[24] S. M. Foiles and M. S. Daw, J. Mater. Res. 2 (1987) 5

[25] H. L. Davis and J. R. Noonan, Phys. Rev. Lett. 54 (1985) 566.

[26] S. P. Chen, A. F. Voter and D. J. Srolovitz, Phys. Rev. Lett. 52 (1986) 1308.

[27] D. Sondericker, F. Jona and P. M. Marcus, Phys. Rev. B 33 (1986) 900.

[28] S. M. Foiles, Surf. Sci. 191 (1987) 329.

[29] Q. Whang, Y. S. Li, C. K. C. Lok, J. Quinn, F. Jona and P. M. Marcus, Surf. Sci. 62 (1987) 181.

[30] T. M. Buck, G. H. Wheatley and L. Marchut, Phys. Rev. Lett. 51 (1983) 43.

[31] W. E. Wallace and G. J. Ackland, Surf. Sci. Lett. 275 (1992) L685.

[32] M. Lundberg, Phys. Rev. B 36 (1987) 4692.

[33] S. M. Foiles, Phys. Rev. B 32 (1985) 7685. 
[34] T. Sakurai, T. Hashizume, A. Jimbo, A. Sakai and S. Hyodo, Phys. Rev. Lett. 55 (1985) 5974; Y. S. Ng, T. T. Tsong and S. B. McLane, Jr., Phys. Rev. Lett. 42 (1979) 588; ibid. Surf. Sci. 84 (1979) 31; Y. S. Ng, S. B. McLane, Jr. and T. T. Tsong, J. Vac. Sci. Technol. 17 (1980) 154; K. Watanabe, M. Hashiba and T. Yamashina, Surf. Sci. 61 (1976) 483; P. R. Webber, C. E. Rojas, P. J. Dobson anbd D. Chadwick, Surf. Sci. 105 (1981) 20; H. H. Brongersma and T. M. Buck, Surf. Sci. 53 (1975) 649; H. H. Brongersma, M. J. Sparnay and T. M. Buck, ibid. 71 (1978) 657; J. Eymery and J. C. Joud, Surf. Sci. 231 (1990) 419;

[35] K. S. Lee, S. H. Kim, H. G. Min, J. Seo and J. S. Kim, Surf. Sci. 377-379 (1997) 918; A. Christensen, A. V. Ruban, P. Stoltze, K. W. Jacobsen, H. L. Skriver, J. K. Norskov and F. Besenbacher, PRB56(1997) 5822.

[36] J. L. Stevens and R. Q. Hwang, Phys. Rev. Lett. 74 (1995) 2078-2081; S. Robert, S. Gauthier, F. Bocquet, S. Rousset, J.L. Duvault and J. Klein, Surf. Sci. 350 (1996)136-144; C. Engdahl, P. Stoltze, K. W. Jacobsen, J. K. Norskov, H. L. Skriver, J. Vac. Sci. Technol. A12 (1994) 17871789; R. K. Schulze, T. N. Taylor and M. T. Paffett, J. Vac. Sci. Technol. A 12 (1994) 3054-3061. [37] R. Darolia, W. S. Walston and M. V. Nathal, Superalloys 1996, R. D. Kissinger et al. eds., The Minerals, Metals and Materials Society, 1996, pp. 561.

[38] G. Bozzolo, C. Amador, J. Ferrante and R. D. Noebe, Scripta Metall. 33 (1995) 1907.

[39] G. Bozzolo, R. D. Noebe, J. Ferrante, A. Garg and C. Amador, to be published.

[40] See, for example, O. K. Andersen, A. V. Postnikov and S. Y. Savrasov, Mat. Res. Soc. Symp. Proc. 253 (1992) 37.

[41] P. Hohenberg and W. Kohn, Phys. Rev. 136 (1964) B864; W. Kohn and L. J. Sham, ibid. 140 (1965) A1133.

[42] G. Bozzolo, Ronald D. Noebe, J. Ferrante and C. Amador, J. Computer-Aided Mater. Design (in press).

[43] J. P. Roux and H. J. Grabke, Appl. Surf. Sci. 68 (1993) 49.

[44] S. A. Chambers, Phys. Rev. B 42 (1990) 10865.

[45] W. D. Ross, J. du Plessis, G. N. van Wyk, E. Taglauer and S. Wolf, J. Vac. Sci. Technol. A 14 (1996) 1648.

[46] R. D. Field, R. Darolia and D. F. Lahrman, Scripta Metall. 23 (1989) 1469.

[47] A. W. Wilson, J. M. Howe, A. Garg and R. D. Noebe in 'Microscopy and Microanalysis 96', ed. G. W. Bailey, San Francisco Press Inc. (1996) 230. 
[48] D. R. Johnson, X. F. Chen, B. F. Oliver, R. D. Noebe and J. D. Whittenberger, Intermetallics 3 (1995) 99.

[49] G. Bozzolo, R. D. Noebe, J. Ferrante and A. Garg, Mat. Sci. Eng. A 239-240 (1997) 769.

[50] G. Bozzolo, R. D. Noebe, J. Ferrante and A. Garg, in Structural Intermetallics 1997, eds. M. V. Nathal, R. Darolia, R. Wagner and M. Yamaguchi, The Mineral, Metals and Materials Society, Warrendale, PA 1997.

[51] R. D. Choudhuri and A. Miller, in Surface Segregation Phenomena, eds. P. A. Dowben, A. Miller, CRC Press, Boca Raton, 1991. 
Public reporting burden for this collection of information is estimated to average 1 hour per response, including the time for reviewing instructions, searching existing data sources, gathering and maintaining the data needed, and completing and reviewing the collection of information. Send comments regarding this burden estimate or any other aspect of this collection of information, including suggestions for reducing this burden, to Washington Headquarters Services, Directorate for Information Operations and Reports, 1215 Jefferson Davis Highway, Suite 1204, Arlington, VA 22202-4302, and to the Office of Management and Budget, Paperwork Reduction Project (0704-0188), Washington, DC 20503. \begin{tabular}{|l|l|l}
\hline 1. AGENCY USE ONLY (Leave blank) & $\begin{array}{c}\text { 2. REPORT DATE } \\
\text { July } 1999\end{array}$ & $\begin{array}{c}\text { 3. REPORT TYPE AND DATES COVERED } \\
\text { Technical Memorandum }\end{array}$
\end{tabular}

4. TITLE AND SUBTITLE 5. FUNDING NUMBERS

Surface Segregation in Multicomponent Systems: Modeling of Surface Alloys and Alloy Surfaces

6. AUTHOR(S)

Guillermo Bozzolo, John Ferrante, Ronald D. Noebe, Brian Good,

Frank S. Honecy, and Phillip Abel

\section{PERFORMING ORGANIZATION NAME(S) AND ADDRESS(ES)}

National Aeronautics and Space Administration

John H. Glenn Research Center at Lewis Field

Cleveland, Ohio 44135-3191

WU-523-22-13-00

National Aeronautics and Space Administration

Washington, DC 20546-0001

8. PERFORMING ORGANIZATION REPORT NUMBER

E-11569

\section{SPONSORING/MONITORING AGENCY NAME(S) AND ADDRESS(ES)}

10. SPONSORING/MONITORING AGENCY REPORT NUMBER

NASA TM-1999-209042

\section{SUPPLEMENTARY NOTES}

Guillermo Bozzolo, Ohio Aerospace Institute, Cleveland, Ohio 44142; John Ferrante, Physics Department, Cleveland State University, Cleveland, Ohio 44115; Ronald D. Noebe, Brian Good, Frank S. Honecy, and Phillip Abel, NASA Glenn Research Center. Responsible person, Guillermo Bozzolo, organization code 5140, (216) 433-5824.

12a. DISTRIBUTION/AVAILABILITY STATEMENT

12b. DISTRIBUTION CODE

Unclassified - Unlimited

Subject Category: 26

Distribution: Nonstandard

This publication is available from the NASA Center for AeroSpace Information, (301) 621-0390.

\section{ABSTRACT (Maximum 200 words)}

The study of surface segregation, although of great technological importance, has been largely restricted to experimental work due to limitations associated with theoretical methods. However, recent improvements in both first-particle and semi-empirical methods are opening the doors to an array of new possibilities for surface scientists. We apply one of these techniques, the BFS method for alloys, which is particularly suitable for complex systems, to several aspects of the computational modeling of surfaces and segregation, including alloy surface segregation, structure and composition of alloy surfaces, and the formation of surface alloys. We conclude with the study of complex NiAl-based binary, ternary and quaternary thin films (with $\mathrm{Ti}, \mathrm{Cr}$ and $\mathrm{Cu}$ additions to $\mathrm{NiAl}$ ). Differences and similarities between bulk and surface compositions are discussed, illustrated by the results of Monte Carlo simulations. For some binary and ternary cases, the theoretical predictions are compared to experimental results, highlighting the accuracy and value of this developing theoretical tool.

14. SUBJECT TERMS

Surface segregation; Alloys; Computational materials science

\begin{tabular}{|c|c|c|}
\hline $\begin{array}{c}\text { 17. SECURITY CLASSIFICATION } \\
\text { OF REPORT } \\
\text { Unclassified }\end{array}$ & $\begin{array}{c}\text { 18. SECURITY CLASSIFICATION } \\
\text { OF THIS PAGE } \\
\text { Unclassified }\end{array}$ & $\begin{array}{c}\text { 19. SECURITY CLASSIFICATION } \\
\text { OF ABSTRACT } \\
\text { Unclassified }\end{array}$ \\
\hline
\end{tabular}

